\title{
Facile Conversion of Toxic Cigarette Butts to N,S-Codoped Carbon Dots and Their Application in Fluorescent Film, Security Ink, Bioimaging, Sensing and Logic Gate Operation
}

Rajkumar Bandi, ${ }^{\dagger}$ Neela Priya Devulapalli, ${ }^{\ddagger}$ Ramakrishna Dadigala, ${ }^{\dagger}$ Bhagavanth Reddy Gangapuram, ${ }^{\dagger, \S}$ and Veerabhadram Guttena*,†०

\author{
${ }^{\dagger}$ Department of Chemistry, Osmania University, Hyderabad 500007, Telangana, India \\ ${ }^{\ddagger}$ Environmental Consultancy Division, Ramky Enviro Services Private Limited, Ramky Grandiose, Gachibowli, Hyderabad 500032, \\ India \\ ${ }^{\S}$ Department of Chemistry, PG Center Wanaparthy, Palamuru University, Mahabubnagar 509001, Telangana, India
}

\section{Supporting Information}

\begin{abstract}
The present work is emphasized on converting toxic cigarette butts (CBs) into highly fluorescent N,S-codoped carbon dots by a facile hydrothermal approach and exploring their multiple applications. The as-produced carbon dots (CBCDs) exhibited bright and stable fluorescence with a quantum yield of $26 \%$ and used as a label-free probe for "on-off-on" sequential detection of $\mathrm{Fe}^{3+}$ and ascorbic acid (AA). The fluorescence of CBCDs can be significantly quenched by $\mathrm{Fe}^{3+}$ ions through static quenching and restored upon the subsequent addition of AA due to the reduction of $\mathrm{Fe}^{3+}$ to $\mathrm{Fe}^{2+}$ by AA. This nanoprobe presented great selectivity and excellent sensitivity to $\mathrm{Fe}^{3+}$ and $\mathrm{AA}$ with a detection limit of 0.13 and $0.2 \mu \mathrm{M}$, respectively. Furthermore, the nanoprobe was extended to biosystem (intracellular detection) and successfully

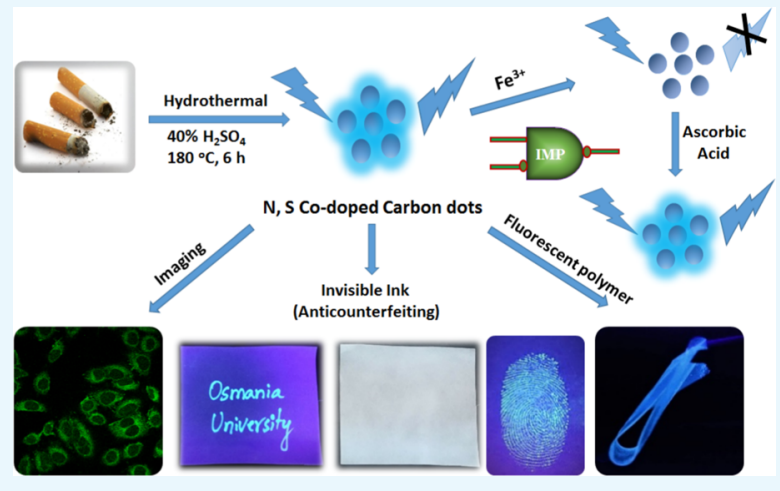
applied for the detection of $\mathrm{Fe}^{3+}$ in real water (tap, bore, and pond) and AA in biological samples (human urine and serum). In addition, we have constructed an IMPLICATION logic gate based on these unique sensing characteristics. The "visible-invisible" and "UV-visible" property explored their use as invisible ink for security applications. Furthermore, highly photostable fluorescent polymer films were prepared by incorporating CBCDs in poly(vinyl alcohol). It is anticipated that the strong and stable fluorescence emission nature of these films might find direct or indirect applications in various optical/optoelectronic devices, ranging from fluorescent displays to light-emitting diodes.
\end{abstract}

\section{INTRODUCTION}

One of the major goals of United Nations Development Programme 2030 is responsible consumption and production of resources. This can be achieved only by efficient management of available resources and proper disposal of toxic waste and pollutants. According to a recent report given by the World Health Organization (WHO 2017), cigarette butts (CBs) and other tobacco wastes make up the major number of individual pieces of litter in the world accounting to more than $40 \%$ of all items collected during urban cleanups. ${ }^{1}$ These butts are washed down by drains and eventually make their way to lakes, rivers, and oceans. Ocean Conservancy also reported similar results in this regard. ${ }^{2}$ Most of these CBs are made up of cellulose acetate, which possess poor photo- and biodegradable properties, making them persistent in the environment. Furthermore, the toxic leachates of CBs pose a severe threat to aquatic life (both marine and freshwater).,4 According to a recent report, CBs are potential sources of nicotine contamination in urban water and can be a major threat to the water quality. ${ }^{5}$ Hence, effective management of these wastes is essential. Conventional disposal methods like landfilling or incineration are neither universally sustainable nor economically feasible for this purpose. ${ }^{6}$

Several researchers have made significant exertions in this concern. Mohajerani et al. made notable contributions by incorporating $\mathrm{CBs}$ in fired clay bricks ${ }^{6}$ and asphalt concrete. These methods offer reduction of large quantities of CBs. However, transforming them into desired products is considered to be a more promising way, as it can offer valueadded products besides waste management. But only a few efforts were made in this regard. Yi et al. ${ }^{8}$ produced highperforming energy-storage material from used cigarette filters. Wei et al. ${ }^{9}$ reported an eco-friendly approach for extracting cellulose acetate from CBs to construct a cellulose-based membrane separator for high-performance lithium-ion batteries. Recently, Yilin et al. ${ }^{10}$ have reported the synthesis of

Received: July 23, 2018

Accepted: October 5, 2018

Published: October 18, 2018 
Scheme 1. Schematic Representation of the Synthesis of Fluorescent CDs from Cigarette Butts

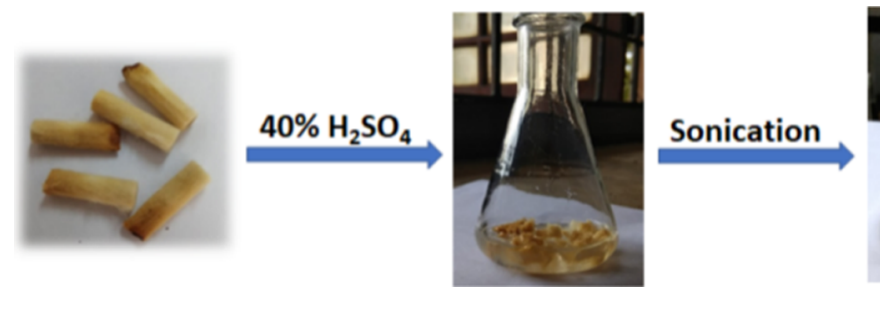

carbon dots (CDs) from cigarette filters and their application for fluorescence detection of Sudan I. However, sufficient characterization data was not presented (chemical composition of formed CDs is not studied) and the possible formation mechanism of CDs (how the water-insoluble cellulose acetate fibers are converted to water-soluble CDs) was not explained.

Fluorescent CDs are a new member of nanocarbon family that comprise discrete, quasi-spherical nanoparticles with sizes below $10 \mathrm{~nm}^{11}$ After their serendipitous discovery from carbon nanotubes, they have gained ever-increasing attention due to their fascinating properties like unique optical properties, multiple functional groups, excellent biocompatibility, and chemical and photostability. ${ }^{12,13}$ They are considered as promising alternatives to fluorescent dyes and quantum dots and are advocated for diversified applications such as sensing, ${ }^{14,15}$ bioimaging, ${ }^{16-18}$ optoelectronic conversion, ${ }^{19,20}$ visible-light-activated bactericide, ${ }^{21}$ fingerprint detection, $^{22}$ dye degradation, ${ }^{23-25}$ solar cells, ${ }^{26}$ printing inks, $^{27}$ and gene delivery. ${ }^{28}$

Preparation methods of CDs reported so far can broadly be grouped into either top-down or bottom-up. The top-down approach involves the breakdown of bulk carbon sources like graphite, carbon nanotubes, and nanodiamonds into fluorescent CDs by employing techniques like arc discharge, laser ablation, chemical oxidation in strong acid, and electrochemical synthesis. ${ }^{11,29}$ Conversely, in the bottom-up approach, the CDs are formed from molecular precursors by applying solvothermal/hydrothermal methods, ${ }^{30-32}$ ultrasound/microwave ${ }^{33-35}$ treatments, or simple thermal combustion. ${ }^{36,37}$ Although a large variety of techniques and starting materials were employed for the production of CDs, the demand for the sustainable synthetic routes that adhere to the principles of green chemistry is still high. To fulfill this requirement, researchers have explored the use of waste materials like food waste, ${ }^{38}$ agriculture waste, ${ }^{39}$ waste paper, ${ }^{40}$ waste frying oil, ${ }^{41}$ etc. as precursors. However, the fluorescence quantum yields (QYs) of the as-produced CDs are less than $10 \%$, limiting the range of their practical applications. Hence, there is a great need of developing facile methods for the largescale utilization of waste resources toward the production of highly fluorescent CDs.

On the other hand, doping CDs with nonmetals such as $\mathrm{N}$, $\mathrm{S}, \mathrm{B}$, and $\mathrm{P}$ received a great scientific attention as it can offer CDs with improved properties like resistance to selfquenching, enhanced fluorescence $\mathrm{QY}$, and sensing selectivity. ${ }^{42}$ Recently, codoping with multiple heteroatoms especially $\mathrm{N}$ and $\mathrm{S}$ has gained extensive consideration because of the improved efficiency resulting from the synergistic effect of the doped $\mathrm{N}$ and $\mathrm{S}$ atoms. ${ }^{43}$

In the present work, we explored the use of CBs as source material for the production of highly fluorescent $\mathrm{N}$ and $\mathrm{S}$ codoped CDs. The as-produced CDs (CBCDs) were systematically characterized by various analytical techniques, and their
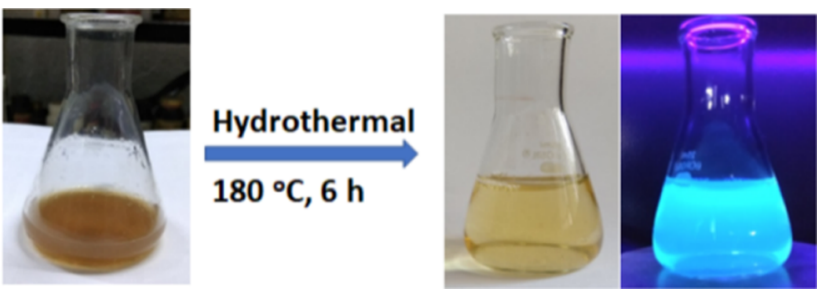

stability toward diverse environmental conditions like high ionic strength, $\mathrm{pH}$, temperature, storage, and continuous irradiation was evaluated. The CBCDs exhibited bright and stable fluorescence with a QY of 26\%. Physicochemical characterizations revealed that the CBCDs are spherical with a mean diameter of $3.7 \pm 1.4$ and composed of graphitic core with amorphous/polar surface functionalities, which impart aqueous solubility. CBCDs were used as "on-off-on" fluorescent probe for the detection of $\mathrm{Fe}^{3+}$ and ascorbic acid (AA). The probe was successfully applied for the analysis of real water (tap, bore, and pond) and biological samples (human urine and serum), and a possible quenching mechanism was proposed. In addition, we have constructed an IMPLICATION logic gate based on these unique sensing characteristics. Excitation-dependent emission behavior coupled with high biocompatibility revealed the multicolor cellular imaging potential of CBCDs. Furthermore, we have prepared fluorescent polymer films by incorporating CBCDs into poly(vinyl alcohol) (PVA). Also these CBCDs were applied as invisible ink for security applications.

\section{RESULTS AND DISCUSSION}

Synthesis of CBCDs. Here, we report a simple hydrothermal method for the production of $\mathrm{N}, \mathrm{S}$ codoped CDs using cigarette butts as source material. The preparation of CDs from CBs is illustrated in Scheme 1. Cigarette butts are mainly composed of cellulose acetate fibers, and they also contain minor amount of chemicals like nicotine, polycyclic aromatic hydrocarbons (PAHs), N-nitrosamine, aromatic amines, and other tarlike components trapped during smoking. ${ }^{6}$ Conversion of cellulose acetate fibers into water-soluble CDs is the critical step. Concentrated sulfuric acid is selected to achieve this conversion due to (1) its strong acid hydrolyzing property, which produces cellulose from cellulose acetate and further facilitates the conversion of cellulose into hydrophilic cellulose nanoparticles; ${ }^{44}$ (2) its strong dehydrating property, which assists the creation of unsaturated $\mathrm{C}=\mathrm{C}$ bonds from saturated $\mathrm{C}-\mathrm{C}$ bonds; and (3) its strong oxidizing property, which facilitates the generation of hydrophilic $\mathrm{C}-\mathrm{O}-\mathrm{H}$ and $\mathrm{O}=\mathrm{C}-$ $\mathrm{O}-\mathrm{H}$ from hydrophobic $\mathrm{C}-\mathrm{H}^{41}$ As $\mathrm{CDs}$ predominantly comprise PAHs in their carbon matrix, ${ }^{45}$ the presence of PAHs in the reaction mixture is expected to facilitate the formation of CDs. Further, the presence of $\mathrm{N}$-containing compounds (nicotine and other aromatic amines) is known to promote the formation of CDs and improve the $\mathrm{QY}$ by $\mathrm{N}$-doping and amine passivation. Furthermore, sulfuric acid can also serve as $S$ dopant to produce $S$ and $\mathrm{N}$-codoped CDs with improved QY. ${ }^{46}$ The crucial role of sulfuric acid is evident from the blank experiment (double-distilled (DD) water is used instead of sulfuric acid), which resulted in CDs with very low QY (3\%) and insignificant production yield. To obtain the best CDs out of waste $\mathrm{CBs}$, process parameters like reaction temperature, 

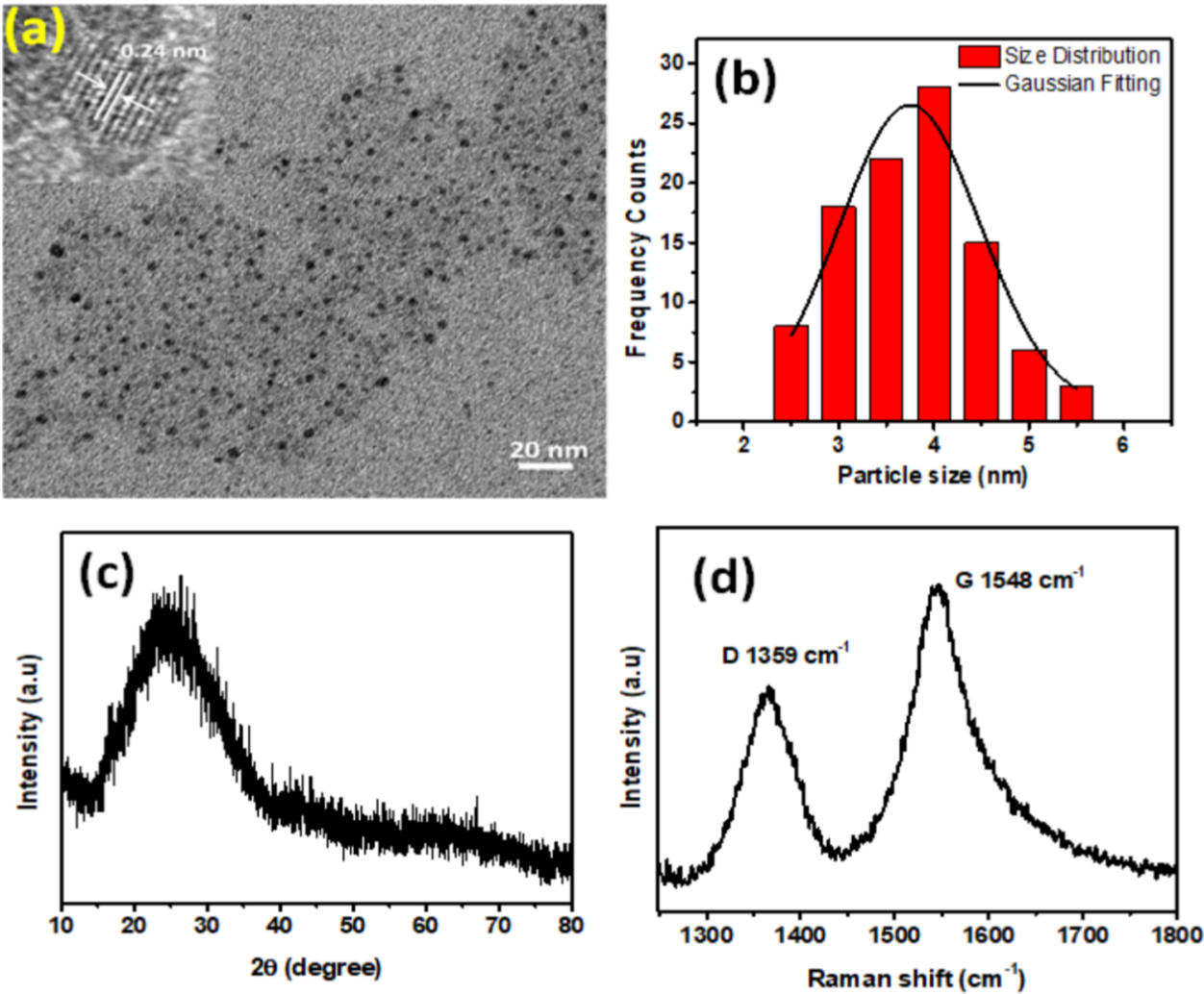

Figure 1. (a) TEM image (inset: HRTEM image), (b) particle size distribution, (c) XRD pattern, and (d) Raman spectrum of CBCDs.
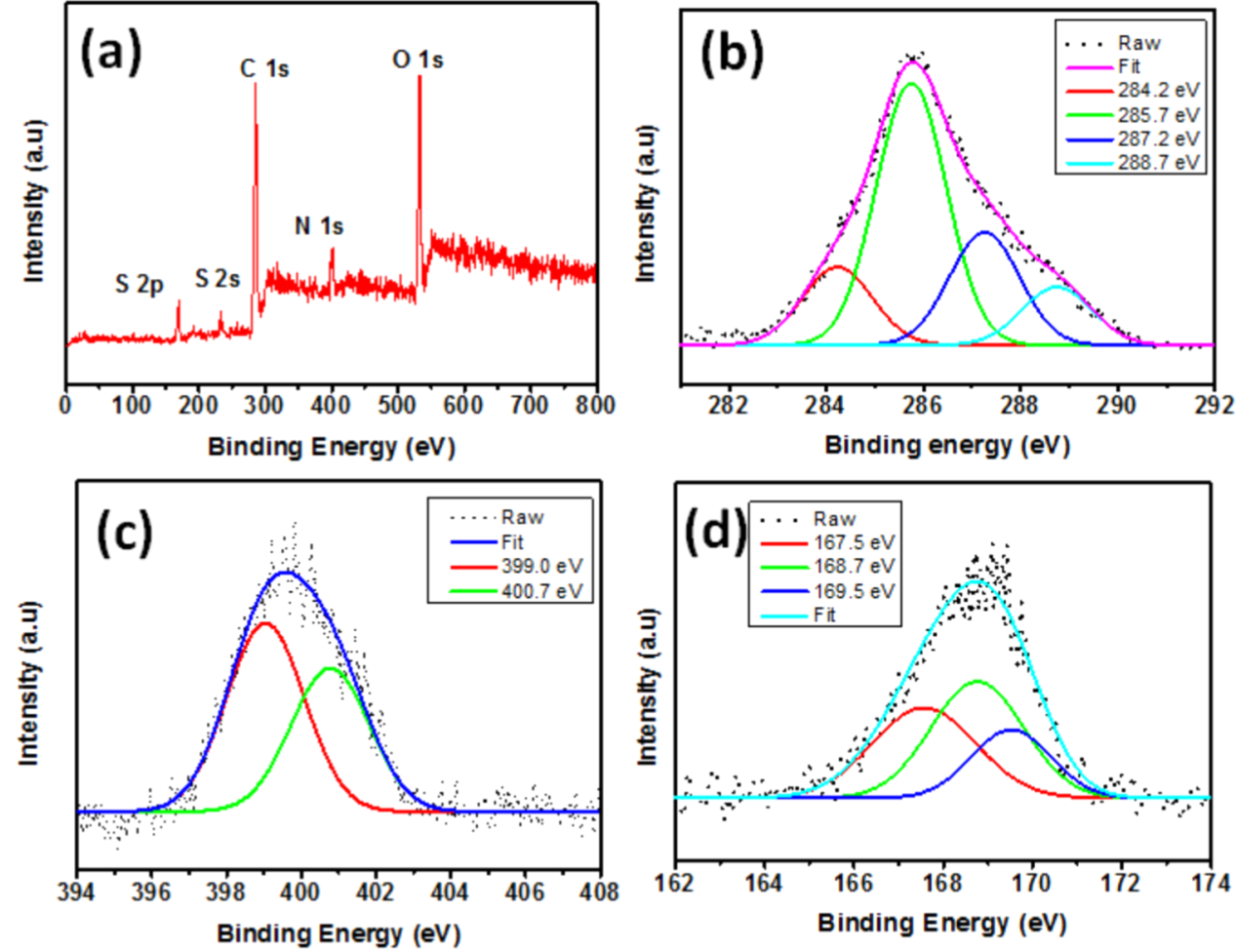

Figure 2. (a) XPS survey spectrum of CBCDs. High-resolution spectra of (b) C 1s, (c) N 1s, and (d) S 2p peaks.

time, and concentration of acid were optimized (Tables $S 1$ and S2). Under optimal conditions, CDs with QY as high as $26 \%$ were obtained with a production yield of $9.6 \%$. A comparison (Table S3) with several previous reports on the production of $\mathrm{CDs}$ from waste materials revealed the advantageous features (mild synthetic conditions and higher QY and production yield) of the present method.

Physicochemical Properties. The structure and morphological features of $\mathrm{CBCDs}$ were explored by transmission electron microscopy (TEM) analysis. As presented in Figure 

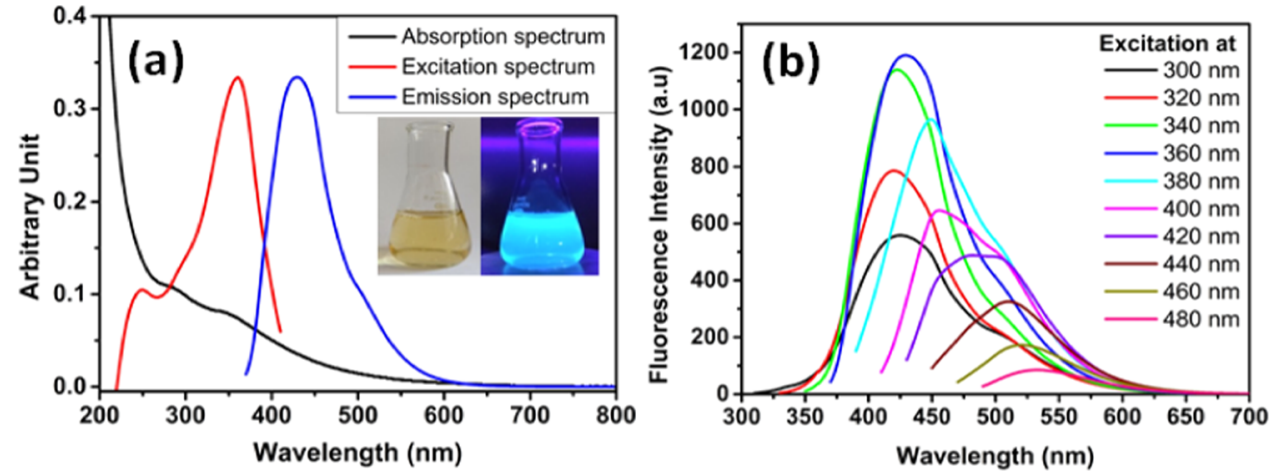

Figure 3. (a) Absorption (black), excitation (red), and emission (blue) spectra of CBCDs (inset: CBCDs aqueous solution under daylight (left) and UV light (right)). (b) Excitation wavelength-dependent emission spectra of CBCDs.

1a, CBCDs possess spherical morphology, are uniform in size, and are well separated from each other. The corresponding size distribution histogram obtained by counting 100 nanoparticles is represented in Figure $1 \mathrm{~b}$. Gaussian fitting of the histogram unveiled the statistical diameter of CBCDs as $3.7 \pm 1.4$. Highresolution TEM (HRTEM) image (inset of Figure 1a) revealed that most of the CBCDs have clear lattice structure with a fringe spacing of $0.24 \mathrm{~nm}$, which agrees with the basal spacing of (1120) lattice plane of graphene. ${ }^{47}$ Powder X-ray diffraction (XRD) patterns of CBCDs (shown in Figure 1c) presented a single broad peak centered at $2 \theta=24.3$ indexed to the (002) lattice spacing of graphitic carbon, indicating the graphite-like structure of CBCDs. The interlayer spacing $d$ $(0.37 \mathrm{~nm})$ is larger than that of graphite $(0.34 \mathrm{~nm})$, articulating the incomplete graphitization and can be attributed to the introduction of oxygen-containing functional groups or owing to the existence of organic functional groups on the surface. ${ }^{48}$ Graphitization is further confirmed by Raman analysis, which exhibited two broad peaks (Figure 1d) centered at 1359 and $1548 \mathrm{~cm}^{-1}$ corresponding to the D and G bands, respectively. The $\mathrm{D}$ band is a measure of disorder in the graphite lattice in the sense that it corresponds to the $\mathrm{A}_{1 \mathrm{~g}}$ (zone-edge) breathing vibration phonon that is only activated in the presence of a neighboring $\mathrm{sp}^{3}$ defect. ${ }^{49}$ The $\mathrm{G}$ band corresponds to the $\mathrm{E}_{2 \mathrm{~g}}$ mode of graphite and is related to the vibration of $\mathrm{sp}^{2}$-bonded carbon atoms in a two-dimensional hexagonal lattice. ${ }^{50}$ The integrated intensity ratio of $\mathrm{D}$ and $\mathrm{G}$ bands $\left(I_{\mathrm{D}} / I_{\mathrm{G}}\right)$, which is characteristic of the extent of disorder and functionalization (ratio of $\mathrm{sp}^{3} / \mathrm{sp}^{2}$ carbons), is found to be 0.66 , signifying the partial disordered graphite-like structure of CBCDs. ${ }^{41}$

The elemental composition of CBCDs revealed by scanning electron microscopy-energy-dispersive X-ray (SEM-EDX) (Figure S1a) is found to be $\mathrm{C}(64.70 \%), \mathrm{N}$ (8.37\%), O (25.27\%), and $S(1.66 \%)$. The surface functional groups of CBCDs were characterized through Fourier transform infrared (FTIR) spectroscopy (Figure S1b). The broad vibrational band in the range of $3600-3100 \mathrm{~cm}^{-1}$ can be attributed to the stretching vibrations of $\mathrm{N}-\mathrm{H}$ and $\mathrm{O}-\mathrm{H}$. A strong band at $1641 \mathrm{~cm}^{-1}$ is attributed to the stretching modes of $\mathrm{C}=\mathrm{N} / \mathrm{C}=$ $\mathrm{O}$ stretching vibration. The peaks at 1568 and $1375 \mathrm{~cm}^{-1}$ can be assigned to $\mathrm{C}=\mathrm{C}$ bond stretching and $\mathrm{C}-\mathrm{H}$ vibrations, respectively. ${ }^{11}$ The vibrational band at $1047 \mathrm{~cm}^{-1}$ is assigned to the presence of $-\mathrm{SO}_{3}{ }^{-}, \mathrm{C}-\mathrm{O}-\mathrm{C}$, and $\mathrm{C}-\mathrm{O}$ bonds. The peak at $1313 \mathrm{~cm}^{-1}$ can be due to $\mathrm{C}\left(\mathrm{sp}^{2}\right)-\mathrm{N}$ and $\mathrm{C}-\mathrm{S}$ bonds, ${ }^{42}$ while the peak at $1155 \mathrm{~cm}^{-1}$ is ascribed to $\mathrm{C}-\mathrm{O}, \mathrm{C}-\mathrm{N}$, and $\mathrm{C}-\mathrm{S}$ bonds. ${ }^{14}$ Further information regarding the nature of bonds and chemical composition of CBCDs was obtained from
X-ray photoelectron spectroscopy (XPS). The XPS survey spectrum (Figure 2a) of CBCDs presented four peaks at 168 , 285,400 , and $532 \mathrm{eV}$, which are ascribed to $\mathrm{S} 2 \mathrm{p}, \mathrm{C} 1 \mathrm{~s}, \mathrm{~N} 1 \mathrm{~s}$, and $\mathrm{O} 1 \mathrm{~s}$ signals, respectively. The high-resolution $\mathrm{C} 1 \mathrm{~s}$ spectrum (Figure $2 \mathrm{~b}$ ) reveals four types of carbon bonds: $\mathrm{sp}^{3}$ $(\mathrm{C}-\mathrm{C}$ and $\mathrm{C}-\mathrm{H})$ at $284.2 \mathrm{eV}, \mathrm{sp}^{2}(\mathrm{C}=\mathrm{C})$ at $285.7 \mathrm{eV}, \mathrm{C}-\mathrm{S} /$ $\mathrm{C}-\mathrm{N} / \mathrm{C}-\mathrm{O}$ at $287.2 \mathrm{eV}$, and $\mathrm{C}=\mathrm{O} / \mathrm{C}=\mathrm{N}$ at $288.7 \mathrm{eV}^{47}$ High-resolution $\mathrm{N} 1 \mathrm{~s}$ spectrum (Figure 2c) designates the existence of pyridinic $\mathrm{N} \mathrm{s}, 1^{\circ} / 2^{\circ}$ amino $\mathrm{N} \mathrm{s}(399.0 \mathrm{eV})$, and pyrrolic N s $(400.7 \mathrm{eV})$. S 2p spectrum (Figure $2 \mathrm{~d}$ ) can be resolved into three distinct peaks at $167.5,168.7$, and $169.5 \mathrm{eV}$, which designates the existence of $\mathrm{C}-\mathrm{SO}_{x}(x=2,3,4)$ on the surface of CBCDs. ${ }^{42} \zeta$ potential of the as-prepared CBCDs suspension was found to be $-14.9 \mathrm{mV}$, demonstrating the negative surface charge of CBCDs (Figure S2). All of these results manifested the occurrence of multiple functional groups like $-\mathrm{OH},-\mathrm{C}=\mathrm{O},-\mathrm{COOH}$, and $-\mathrm{NH}$ and successful doping of $\mathrm{N}, \mathrm{S}$ elements in the $\mathrm{CB}$-derived CDs.

Optical Properties. The UV-vis absorption, excitation, and emission spectra are represented in Figure 3a. As shown in the figure, the absorption spectrum displays two poorly resolved bands around 270 and $320 \mathrm{~nm}$, which can be ascribed to the $\pi-\pi^{*}$ transition of carbonic core center and $\mathrm{n}-\pi^{*}$ transition of heteroatomic surface functionalities or molecule center, respectively. ${ }^{51}$ Similar to several previous reports, aqueous solution of $\mathrm{CBCDs}$ exhibit bright blue emission under $365 \mathrm{~nm}$ UV light, and it appeared transparent and pale yellow under daylight (inset of Figure $3 \mathrm{a}$ ). The corresponding excitation spectrum shows two peaks, which can be ascribed to core and surface excitations. Like most of the fluorescent CDs, CBCDs also exhibited excitation tunable emission behavior. A steady increase in $\lambda_{\text {ex }}$ from 300 to $480 \mathrm{~nm}$ resulted in a red shift in the emission peak position along with a concurrent first increase and then decrease in the emission intensity (Figure $3 \mathrm{~b}$ ). This red shift can be clearly observed in the corresponding normalized emission spectra (Figure S3). This tunable emission is considered to be the versatile characteristic of CDs and has been most prominently ascribed to the selective excitation of subsets of CDs within the $\mathrm{CD}$ ensemble. ${ }^{52}$ The maximum emission peak centered at $430 \mathrm{~nm}$ was observed under the excitation of $360 \mathrm{~nm}$ with a large stokes shift of $70 \mathrm{~nm}$. As presented in Figure S4, the fluorescence $\mathrm{QY}$ of $\mathrm{CBCDs}$ at room temperature was calculated to be $26 \%$ (using quinine sulfate (QS) as reference), which is greater compared to various previous reports (Table S3). The reason for this higher QY may be due to the synergistic effect of doped $\mathrm{N}$ and $\mathrm{S}$ atoms. 

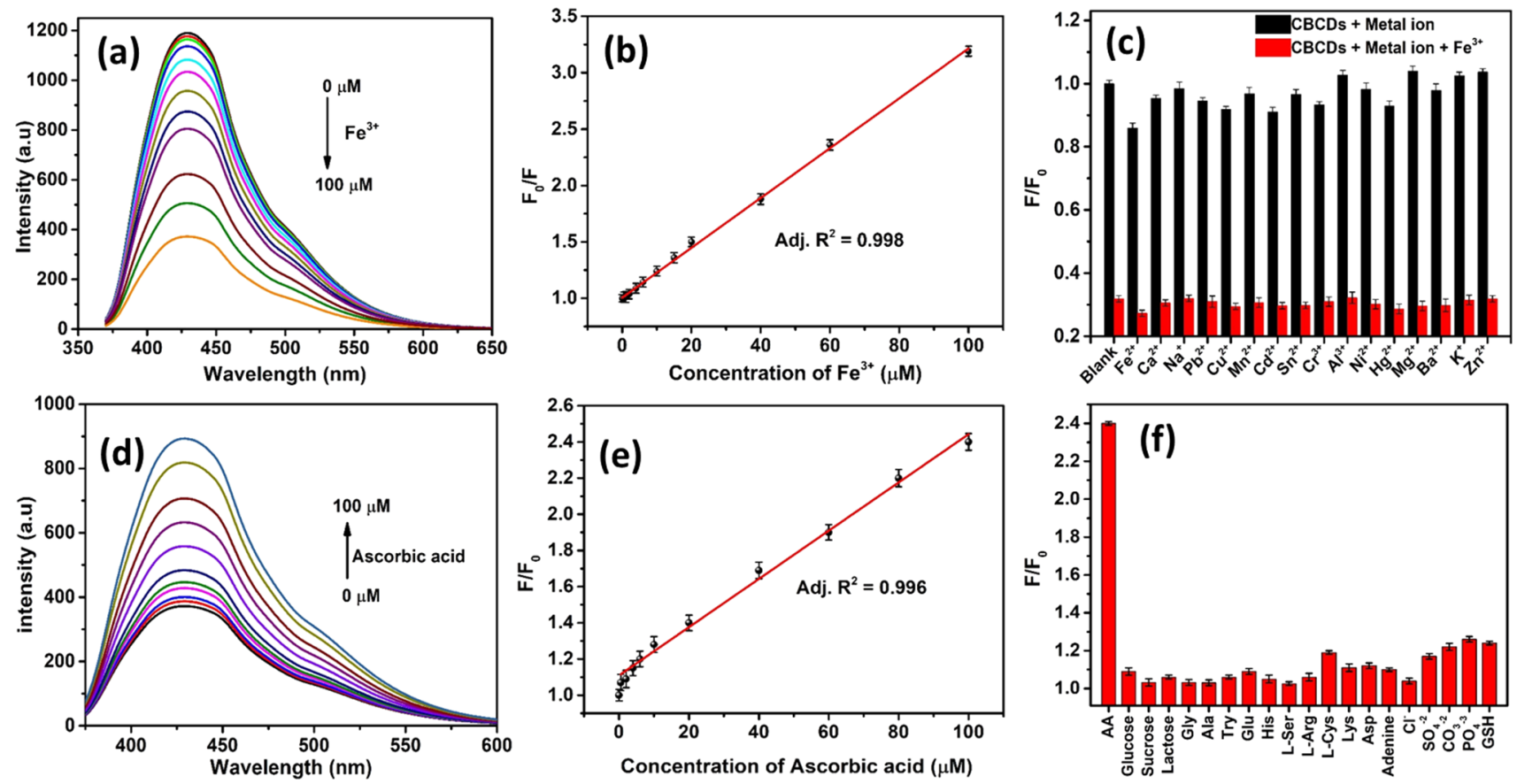

Figure 4. (a) Fluorescence intensity response of $\mathrm{CBCDs}$ with increasing concentration of $\mathrm{Fe}^{3+}$. (b) Relationship between $F_{0} / F$ and the concentration of $\mathrm{Fe}^{3+}$ ions in the range of $0-100 \mu \mathrm{M}(0,0.5,1,2,4,6,10,15,20,40,60,100 \mu \mathrm{M})$. (c) Fluorescence response $\left(F / F_{0}\right)$ of $C B C D s$ toward various metal ions (black) and subsequent addition of $\mathrm{Fe}^{3+}$ ions (red). (d) Fluorescence intensity response of $\mathrm{CBCDs} / \mathrm{Fe}^{3+}$ with increasing concentration of AA. (e) Relationship between $F / F_{0}$ and the concentration of AA in the range of $0-100 \mu \mathrm{M}(0,0.5,2,4,6,10,20,40,60,80,100$ $\mu \mathrm{M})$. (f) Fluorescence response $\left(F / F_{0}\right)$ of $\mathrm{CBCDs} / \mathrm{Fe}^{3+}$ system toward various commonly interfering species.

Stability. In view of the fact that fluorescence emission of many fluorescent probes can be disrupted by the complex environmental conditions, we have examined the emission spectra of CBCDs under diverse conditions to verify their practical applicability. The effect of ionic strength was examined by recording the emission spectra under various $\mathrm{KCl}$ concentrations. As depicted in Figure S5a, only a slight diminution in the photoluminescence (PL) intensity is observed even at a high $\mathrm{KCl}$ concentration of $2 \mathrm{M}$, which verifies the excellent stability of $\mathrm{CBCDs}$ under high-ionicstrength environments and outspreads their usage to identical ion-rich biological conditions. As shown in Figure S5b, change in the solution $\mathrm{pH}$ did not alter the peak position, but caused fluctuations in the emission intensity. No significant alteration in the emission intensity is recorded over a $\mathrm{pH}$ range of 5-9, which is a beneficial property for the fluorescent probe to be used in complex biological system and practical applications. Photostability studies revealed their excellent property of resistance to photobleaching (Figure $\mathrm{S5c}$ ). As presented in Figure S5d, the fluorescence intensity of CBCDs was found almost stable in the temperature range of $25-45{ }^{\circ} \mathrm{C}$ representing their thermal stability. Further, the storage stability of CBCDs was examined by keeping those under (1) ambient conditions and (2) refrigerator for 60 days. Virtually no fluctuations in their emission intensity and no obvious precipitation were observed in both the cases (Figure S6), demonstrating their great stability and long shelf life.

Detection of $\mathrm{Fe}^{3+}$ and AA. The aforementioned optical merits, along with the existence of abundant surface functional groups encouraged us to further investigate the possible sensing applications of CBCDs. According to several previous reports, the surface functional groups of $\mathrm{CDs}$ can selectively interact with metal ions and produce a fluorescence change; thus, we have studied the fluorescence response of CBCDs toward various biologically and environmentally important metal ions, such as $\mathrm{Fe}^{2+}, \mathrm{Ca}^{2+}, \mathrm{Na}^{+}, \mathrm{Pb}^{2+}, \mathrm{Cu}^{2+}, \mathrm{Mn}^{2+}, \mathrm{Cd}^{2+}$, $\mathrm{Sn}^{2+}, \mathrm{Cr}^{3+}, \mathrm{Al}^{3+}, \mathrm{Ni}^{2+}, \mathrm{Hg}^{2+}, \mathrm{Mg}^{2+}, \mathrm{Ba}^{2+}, \mathrm{K}^{+}, \mathrm{Zn}^{2+}$, and $\mathrm{Fe}^{3+}$. As shown in Figure S7, among all ions, only $\mathrm{Fe}^{3+}$ caused a severe decline in the PL intensity, which unveiled the applicability of CBCDs as highly selective turn-off fluorescent probes for $\mathrm{Fe}^{3+}$ detection. This discrimination effect for $\mathrm{Fe}^{3+}$ can be credited to the special coordination between electron-deficient $\mathrm{Fe}^{3+}$ ions and electron-rich surface functional groups of $\mathrm{CBCDs} .{ }^{46}$ In the course of developing an efficient probe, some critical parameters, including usage concentration of CBCDs, incubation time, and solution $\mathrm{pH}$, were optimized. In general, in the presence of quencher of a given concentration, lower concentration of fluorophore will result in greater sensitivity and higher concentration will achieve a broader detection range; ${ }^{53}$ hence, through comprehensive consideration of both sensitivity (limit of detection (LOD)) and linear detection range, $0.05 \mathrm{mg} \mathrm{mL}^{-1}$ of $\mathrm{CBCDs}$ was selected as optimum. Quenching kinetic investigations (Figure S8a) revealed that 2 min of interaction with $\mathrm{Fe}^{3+}$ is sufficient to produce maximum fluorescence response, which remained stable in the following 20 min of observation, suggesting the complexation between $\mathrm{Fe}^{3+}$ and $\mathrm{CBCD}$ is quick and stable, which is useful in rapid sensing without strict time control. ${ }^{54}$ As presented in Figure $\mathrm{S} 8 \mathrm{~b}, \mathrm{Fe}^{3+}$ ions were able to produce decent response in the $\mathrm{pH}$ range of 6-9, which is beneficial as most environmental and biological samples lie in this $\mathrm{pH}$ scale and maximum response is achieved at $\mathrm{pH} 7$, which is taken as optimum.

Under optimal circumstances, sensitivity was inspected by computing the fluorescence response of $\mathrm{CBCDs}$ to several concentrations of $\mathrm{Fe}^{3+}$ in the range of $0-100 \mu \mathrm{M}$. As depicted in Figure $4 \mathrm{a}$, the PL intensity gradually dwindled with 
Scheme 2. Schematic Illustration of On-Off-On Detection of $\mathrm{Fe}^{3+}$ and Ascorbic Acid

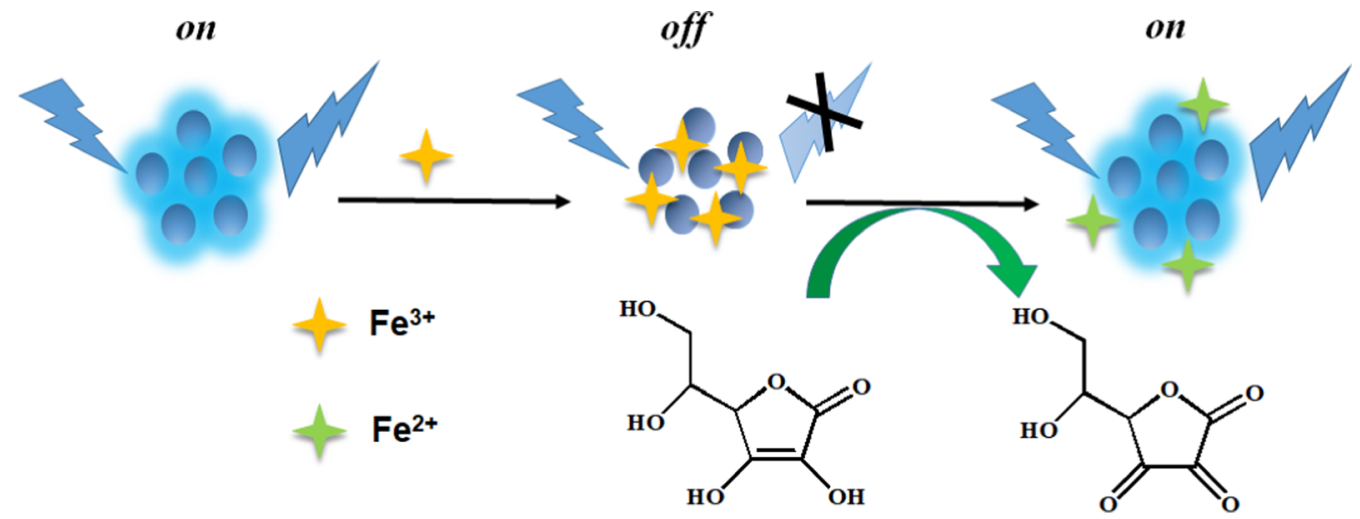

increasing concentrations of $\mathrm{Fe}^{3+}$. The plot of quenching efficiency $\left(F_{0} / F\right)$ versus $\mathrm{Fe}^{3+}$ concentration (Figure $4 \mathrm{~b}$ ) displayed a good linearity $\left(R^{2}=0.998\right)$ in the range of $0-$ $100 \mu \mathrm{M}$, where $F_{0}$ and $F$ are the PL intensities of CBCDs at $430 \mathrm{~nm}$ in the absence and presence of $\mathrm{Fe}^{3+}$, respectively. The detection limit (LOD) was estimated to be $0.13 \mu \mathrm{M}$ based on the equation $3 \sigma / m$, where $\sigma$ is the standard deviation of the blank signal $(n=6)$ and $m$ is the slope of the linear fit. The LOD presented by our method $(0.13 \mu \mathrm{M})$ is much lower than the maximum permissible limit $(5.36 \mu \mathrm{M})$ stipulated by World Health Organization (WHO) and LOD presented by other researchers for $\mathrm{Fe}^{3+}$ in drinking water. ${ }^{55}$ Comparison of analytical performance of CBCDs nanoprobe with several chosen probes in the literature (Table S4) revealed the superiority of the present sensor in terms of linear range, LOD, and applicability to real samples.

With inherent complexity, metal-ion detection in real samples poses a great challenge to the analytical methods not only in terms of sensitivity but more importantly in selectivity. Hence, to further explore the applicability of the proposed nanoprobe to real samples, selectivity and competition experiments were carried out. The black bars shown in Figure $4 c$ depict the PL response $\left(F / F_{0}\right)$ of $C B C D$ s to various metal ions each at a concentration of $200 \mu \mathrm{M}$. It is evident that none of these metal ions caused a considerable decline in the PL intensity. Competition experiments were conducted by subsequently adding $100 \mu \mathrm{M}$ of $\mathrm{Fe}^{3+}$ to the above solutions. As represented by the red bars in Figure 4c, no substantial change in the PL response $\left(F / F_{0}\right)$ appeared in the co-presence of $\mathrm{Fe}^{3+}$ and other metal ions in comparison to that of $\mathrm{Fe}^{3+}$ alone. All of these results clearly demonstrated the excellent selectivity of CBCD-based nanoprobe and motivated us to investigate their practical applicability in real samples.

It is observed that the quenched fluorescence of CBCDs/ $\mathrm{Fe}^{3+}$ can be recovered by the addition of AA. This can be attributed to the (i) antioxidant nature of $\mathrm{AA}$, which can reduce $\mathrm{Fe}^{3+}$ to $\mathrm{Fe}^{2+}$ and (ii) the high selectivity of CBCDs toward $\mathrm{Fe}^{3+}$ over $\mathrm{Fe}^{2+}$. Moreover, $\mathrm{AA}$ did not exhibit any significant effect on the fluorescence of CBCDs alone. Hence, the $\mathrm{CBCDs} / \mathrm{Fe}^{3+}$ system can be employed as a turn on fluorescent probe for the detection of AA (Scheme 2). Kinetic investigations disclosed that, $10 \mathrm{~min}$ of interaction with $\mathrm{AA}$ recovered the emission of $\mathrm{CBCDs} / \mathrm{Fe}^{3+}$ system to a stable value (Figure S9), and this $10 \mathrm{~min}$ is taken as the incubation time for the assay. Sensitivity of $\mathrm{CBCDs} / \mathrm{Fe}^{3+}$ system toward $\mathrm{AA}$ is evaluated by recording its fluorescence response to various concentrations of AA. As presented in Figure 4d, the emission intensity of $\mathrm{CBCDs} / \mathrm{Fe}^{3+}$ nicely recovered with increase in the concentration of $\mathrm{AA}$, and the plot of $F / F_{0}$ versus AA concentration (Figure $4 \mathrm{e}$ ) demonstrated a good linearity $\left(R^{2}=0.996\right)$ in the range of $0.5-100 \mu \mathrm{M}$, where $F_{0}$ and $F$ are the emission intensities of $\mathrm{CBCDs} / \mathrm{Fe}^{3+}$ at $430 \mathrm{~nm}$ in the absence and presence of $\mathrm{AA}$, respectively. The detection limit (LOD) was estimated to be $0.2 \mu \mathrm{M}$ based on the equation $3 \sigma / m$, where $\sigma$ is the standard deviation of the blank signal $(n=6)$ and $m$ is the slope of the linear fit. Selectivity of the probe is evaluated by studying the interference of various amino acids, anions, and sugars. As depicted in Figure 4f, the probe presented greater selectivity, which opened their further applicability to real samples. The sensing performance (linear range and limit of detection) of the present probe is compared to that of several reported methods (Table S5), which revealed that the presented method is among the best methods reported so far.

Analysis of Real Samples. Practical applicability of CBCDs nanoprobe was assessed by detecting $\mathrm{Fe}^{3+}$ in real water (tap, bore, and pond) and AA in complex biological (human urine and serum) samples. To avoid the particulate matter, real water samples are subjected to centrifugation and filtration $(0.45 \mu \mathrm{m}$ membrane filter $)$. Serum and urine samples are subjected to a 100-fold dilution with PBS before analysis. Then, the water samples were spiked with various concentrations of standard $\mathrm{Fe}^{3+}$ solution, and the serum and urine samples were spiked with AA and analyzed by the proposed method. As shown in Tables S6 and S7, good recoveries (98$102)$ and high analytical precision with RDS $3.2(n=6)$ were obtained. Furthermore, the nanoprobe is validated by comparing the results of $\mathrm{Fe}^{3+}$ and $\mathrm{AA}$ detection in a real sample to those of a standard method (atomic absorption spectrometry for $\mathrm{Fe}^{3+}$ and high-performance liquid chromatography-UV for AA). These results confirm the reliability and feasibility of the proposed nanosensor for monitoring $\mathrm{Fe}^{3+}$ in environmental and AA in biological samples.

Possible Mechanism of Fluorescence Quenching. In general, several sorts of molecular interactions between the fluorophore and quencher such as electron or energy transfer, excited-state reaction, collisional quenching, and ground-state complex formation can lead to fluorescence quenching. ${ }^{56}$ The quenching mechanisms are typically classified into (1) dynamic quenching, involving the transfer of electron from excited-state fluorophore to ground-state quencher by means of collision and (2) static quenching resulting from the formation of a nonfluorescent ground-state complex between the fluorophore and quencher. 

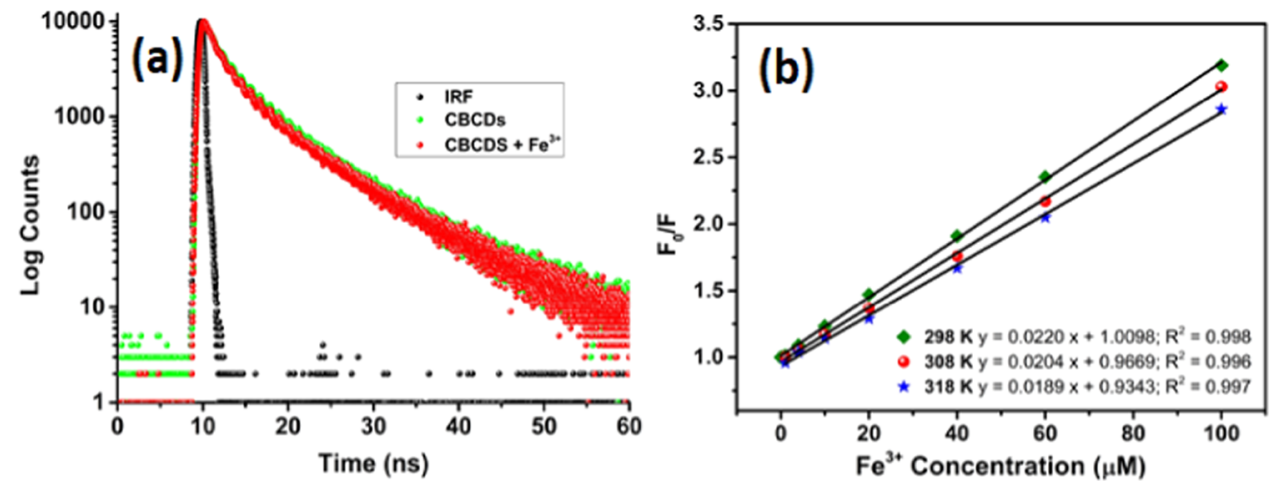

Figure 5. (a) Fluorescence decay of CBCDs in the absence (green) and presence (red) of $\mathrm{Fe}^{3+}$ ions. (b) Stern-Volmer plots $\left(F_{0} / F\right.$ vs $\mathrm{Fe}^{3+}$ concentration) at different temperatures.

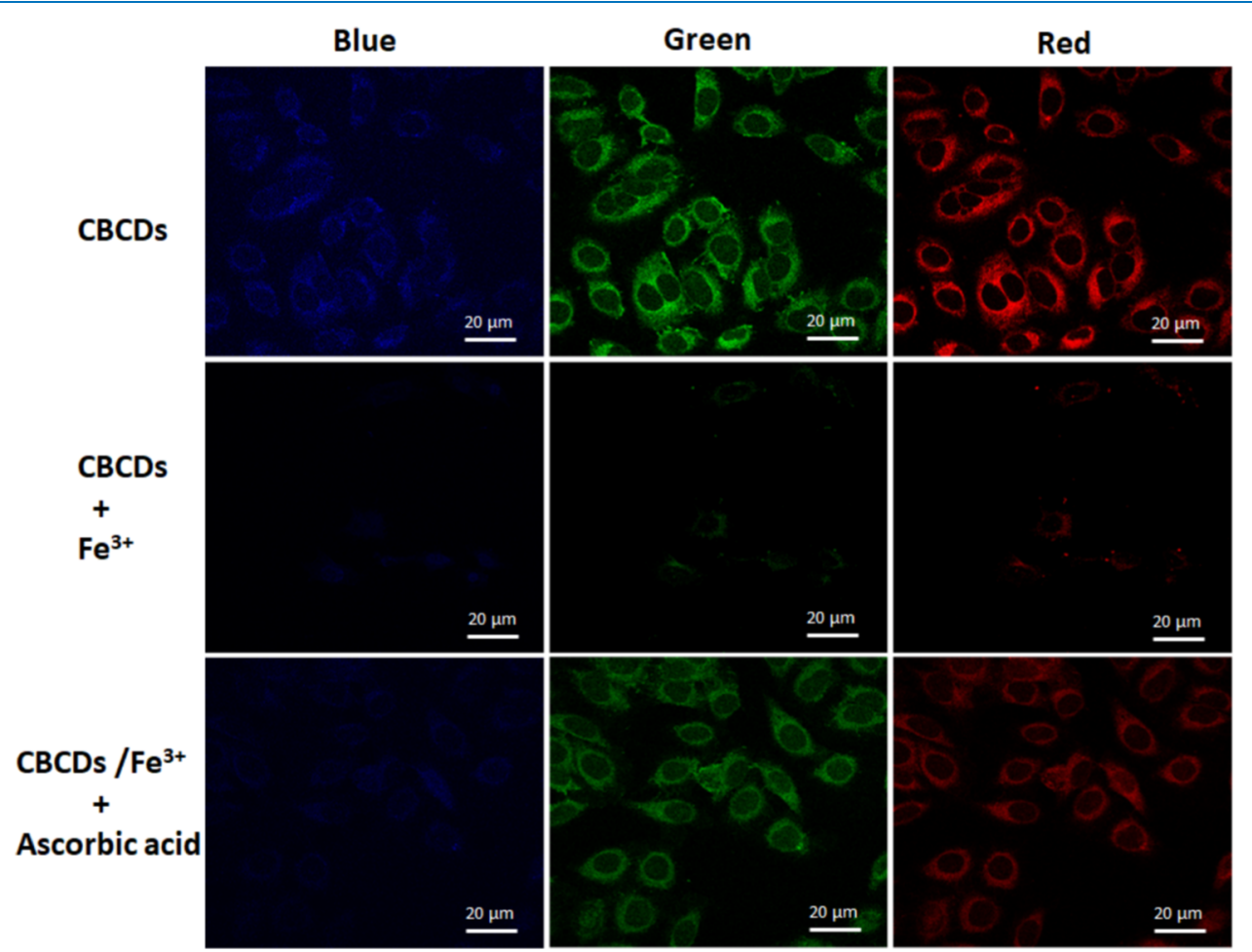

Figure 6. Confocal microscopic images of HeLa cells: cells treated with $200 \mu \mathrm{g} \mathrm{mL} \mathrm{L}^{-1} \mathrm{CBCDs}$ for $6 \mathrm{~h}$; cells (CBCDs treated) further treated with $200 \mu \mathrm{M} \mathrm{Fe}^{3+}$ for $1 \mathrm{~h}$; and cells (CBCDs/Fe $\mathrm{Fe}^{3+}$ treated) further treated with $500 \mu \mathrm{M}$ AA for $1 \mathrm{~h}$, and images taken under three excitation wavelengths of $405 \mathrm{~nm}$ (blue), $488 \mathrm{~nm}$ (green), and $561 \mathrm{~nm}$ (red).

To explore the possible mechanism of quenching of CBCDs fluorescence by $\mathrm{Fe}^{3+}$ ions, the standard Stern-Volmer equation is applied.

$$
\mathrm{F}_{0} / \mathrm{F}=1+K_{\mathrm{sv}}[\mathrm{Q}]=1+k_{\mathrm{q}} \tau_{0}[\mathrm{Q}]
$$

where $F_{0}$ and $F$ refer to the fluorescence intensities of CBCDs in the absence and presence of quencher $\left(\mathrm{Fe}^{3+}\right)$, respectively; $K_{\mathrm{sv}}$ and $k_{\mathrm{q}}$ represent the Stern-Volmer quenching constant and bimolecular quenching constant, respectively; [Q] is the concentration of quencher $\left(\mathrm{Fe}^{3+}\right)$; and $\tau_{0}$ is the average lifetime of CBCDs in the absence of quencher.

As depicted in Figure $4 \mathrm{~b}$, the plot of $F_{0} / F$ versus [Q] exhibited a good linear correlation (correlation coefficient, $R^{2}$ $=0.998)$ and the Stern-Volmer quenching constant $\left(K_{\mathrm{sv}}\right)$ was found to be $2.2 \times 10^{4} \mathrm{M}^{-1}$. The excellent linear correlation infers that the observed quenching is either purely dynamic or purely static and rules out the possibility of static-dynamic combination. ${ }^{51}$ In principle, fluorescence lifetime measurement is the most definitive method to distinguish dynamic and static quenching processes. ${ }^{57}$ In light of this, fluorescence lifetime quenching analysis is carried out to confirm the nature of quenching. Figure 5a reveals that the PL lifetime of CBCDs is not quenched by $\mathrm{Fe}^{3+}$ ions, which rule out the possibility of dynamic quenching. The bimolecular quenching constant $\left(k_{\mathrm{q}}\right)$ calculated from $K_{\mathrm{sv}}$ and average lifetime $\tau_{0}(5.26 \mathrm{~ns})$ is found to be $4.18 \times 10^{13} \mathrm{M}^{-1} \mathrm{~s}^{-1}$, which is higher than the diffusioncontrolled limit $\left(10^{10} \mathrm{M}^{-1} \mathrm{~s}^{-1}\right)$ and further supports the static quenching mechanism involving the ground-state complex formation. Decline in the quenching constant with rise in the temperature (Figure 5b) further supported the static quenching process. All of these findings designate that the quenching caused by $\mathrm{Fe}^{3+}$ ions is a result of nonfluorescent complex formed between the surface functional groups of $\mathrm{CBCDs}$ and $\mathrm{Fe}^{3+}$ ions. FTIR spectral analysis was further used 
(a)

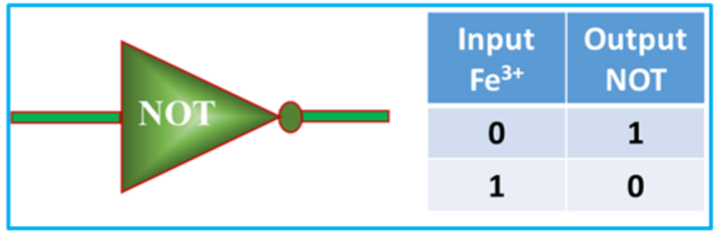

(b) (c)
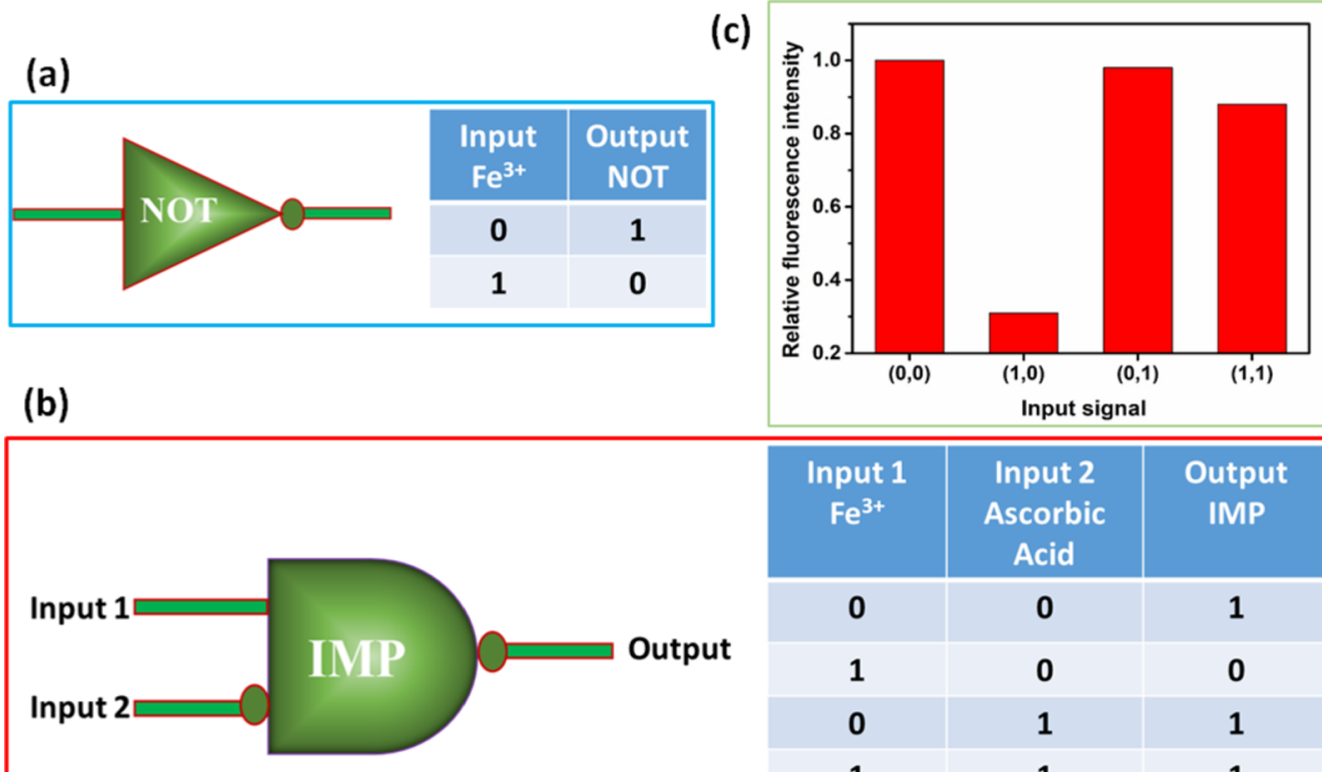

\begin{tabular}{|c|c|c|}
\hline $\begin{array}{c}\text { Input 1 } \\
\mathrm{Fe}^{3+}\end{array}$ & $\begin{array}{c}\text { Input 2 } \\
\text { Ascorbic } \\
\text { Acid }\end{array}$ & $\begin{array}{c}\text { Output } \\
\text { IMP }\end{array}$ \\
\hline $\mathbf{0}$ & $\mathbf{0}$ & $\mathbf{1}$ \\
\hline $\mathbf{1}$ & $\mathbf{0}$ & $\mathbf{0}$ \\
\hline $\mathbf{0}$ & $\mathbf{1}$ & $\mathbf{1}$ \\
\hline $\mathbf{1}$ & $\mathbf{1}$ & $\mathbf{1}$ \\
\hline
\end{tabular}

Figure 7. Logic operations using CBCDs: (a) logic symbol and truth table of NOT logic gate; (b) logic symbol and truth table of IMPLICATION logic gate; and (c) fluorescence response of CBCDs under different inputs.

to verify this assumption. As shown in Figure S10, evident changes (especially at the characteristic peaks of $\mathrm{C}-\mathrm{O}, \mathrm{C}-\mathrm{N}$, and $\mathrm{SO}_{x}^{-}$) were observed in the FTIR spectrum of $\mathrm{CBCDs}$ after the addition of $\mathrm{Fe}^{3+}$, which further confirmed that $\mathrm{Fe}^{3+}$ indeed coordinated with these surface functional groups of CBCDs.

Cytotoxicity and Cellular Imaging. With the grander optical properties like bright fluorescence ( $Q Y=26 \%)$, high ionic strength, and tolerance to photobleaching and complex environmental conditions, the $\mathrm{CBCDs}$ showcase a great potential to serve as bioimaging agents. However, like various biological applications, low cytotoxicity is the key requirement for a material to be used as bioimaging agent also. Hence, we have evaluated the inherent cytotoxicity of CBCDs toward human normal (HEK-293) and human cancerous (HeLa) cells. Standard 3-(4,5-dimethylthiazol-2-yl)-2,5-diphenyltetrazolium bromide (MTT) cell viability assay is employed for this purpose. As shown in Figure S11, CBCDs did not exhibit substantial toxicity on both cancerous and noncancerous cells and the cell viabilities remain $85 \%$ even at a concentration of $1000 \mu \mathrm{g} \mathrm{mL}{ }^{-1}$, denoting their excellent biocompatibility. An important observation is that, at the dosage used for bioimaging $\left(200 \mu \mathrm{g} \mathrm{mL}^{-1}\right)$, virtually no toxicity is witnessed (cell viability is over 95\%), which indicated that the CBCDs have great potential for biomedical applications such as in vivo imaging, cellular labeling, and medical imaging. ${ }^{58}$ These results are in agreement with similar studies carried out earlier. ${ }^{59}$ Further, it is interesting to note that CDs derived from toxic CBs did not exhibit any toxicity toward human normal and cancerous cells demonstrating their versatility.

Cellular imaging potential of CBCDs was evaluated by performing the in vitro cellular uptake experiments with $\mathrm{HeLa}$ cells. After incubating with $200 \mu \mathrm{g} \mathrm{mL}^{-1}$ CBCDs for $6 \mathrm{~h}$, the cells were imaged under a confocal laser scanning microscope. As shown in Figure 6, the cells were brightly illuminated with blue, green, and red colors under the excitation of 408, 488, and $543 \mathrm{~nm}$, respectively. Nevertheless, the control cells did not display any detectable fluorescence under the same exposure conditions. A closer observation of the images revealed that the fluorescence glow is mainly confined to cell membrane and cytoplasmic area while the nucleus had only a weak glow. These results indicate that, similar to some previous reports, ${ }^{60} \mathrm{CBCDs}$ were also having difficulties in labeling the nucleus, while the cytoplasm and cell membrane are easily stained.

Intracellular Detection of $\mathrm{Fe}^{3+}$ and $\mathrm{AA}$. It has been reported that overload and deficiency of $\mathrm{Fe}^{3+}$ can disturb the cellular homeostasis, resulting in various diseases. ${ }^{61}$ Hence, developing a simple and sensitive probe for monitoring intracellular $\mathrm{Fe}^{3+}$ is of great importance. Impressed by the favorable biocompatibility, cell imaging ability, and high selectivity, CBCDs were further applied for monitoring $\mathrm{Fe}^{3+}$ in live cells by introducing exogenous $\mathrm{Fe}^{3+}$ into the $\mathrm{CBCD}$ pretreated HeLa cells. As expected, the confocal microscopic images taken after supplementing cells with $200 \mu \mathrm{M} \mathrm{Fe}^{3+}$ in the growth medium exhibited very weak intracellular fluorescence (Figure 6). Further treatment of cells with AA resulted in the nicely recovered intracellular fluorescence indicating that emission of $\mathrm{CBCDs} / \mathrm{Fe}^{3+}$ in the cells can be recovered by $\mathrm{AA}$. It can be observed that the fluorescence emission of the cells is in the order of CBCDs $>$ CBCDs/ $\mathrm{Fe}^{3+}$ / $\mathrm{AA}>\mathrm{CBCDs} / \mathrm{Fe}^{3+}$ treated. All of these results elucidate that CBCDs could serve as efficient fluorescent probe for "on-offon" detection of $\mathrm{Fe}^{3+}$ and $\mathrm{AA}$ in living cells.

Logic Operations of CBCDs. The fluorescence switching behavior of $\mathrm{CBCD}$ in the presence of $\mathrm{Fe}^{3+}$ and $\mathrm{AA}$ can be employed as multiple molecular logic gates, performing the Boolean algebraic logic operations. ${ }^{62}$ The simple single input molecular logic gate NOT can be constructed using $\mathrm{Fe}^{3+}$ as single input signal (Figure 7a). The multiple input logic gate IMPLICATION can be realized by taking $\mathrm{Fe}^{3+}$ and $\mathrm{AA}$ as input 1 and input 2 , respectively. The presence of $\mathrm{Fe}^{3+}$ or $\mathrm{AA}$ is taken as 1 and their absence as 0 . For output, the maximum fluorescence was taken as 1 and the corresponding quenched fluorescence as 0 . As shown in Figure $7 b$, only in the presence of $\mathrm{Fe}^{3+}$ and absence of $\mathrm{AA}$, i.e., input $(1,0)$, significant 


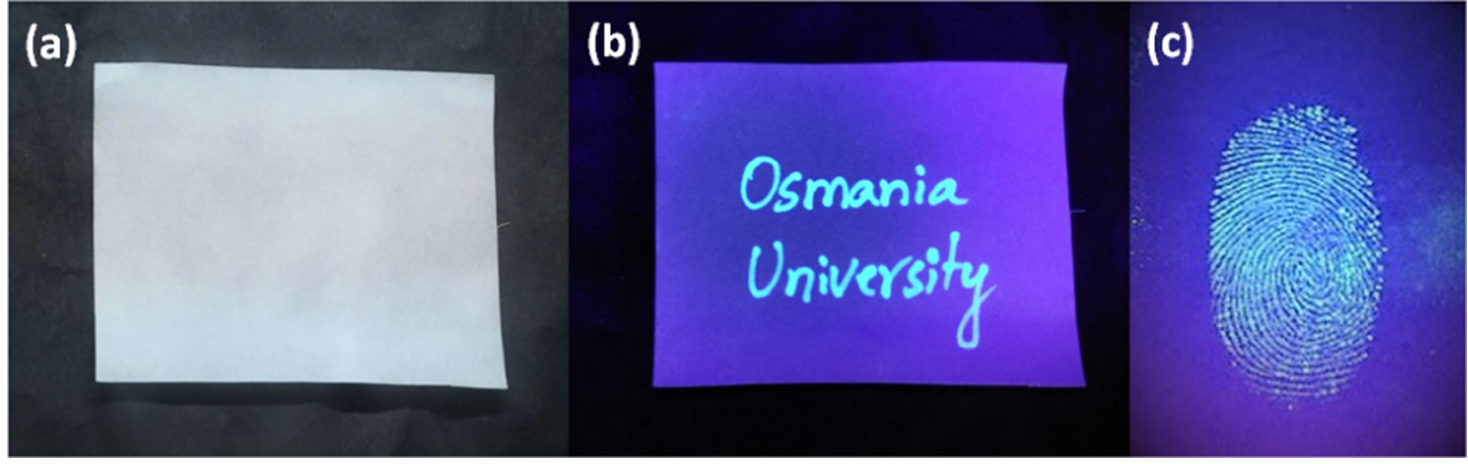

Figure 8. Information loaded on commercial filter paper using CBCDs invisible ink under (a) daylight and (b) $365 \mathrm{~nm}$ UV light. (c) CBCDsformed fingerprints under $365 \mathrm{~nm}$ UV light.

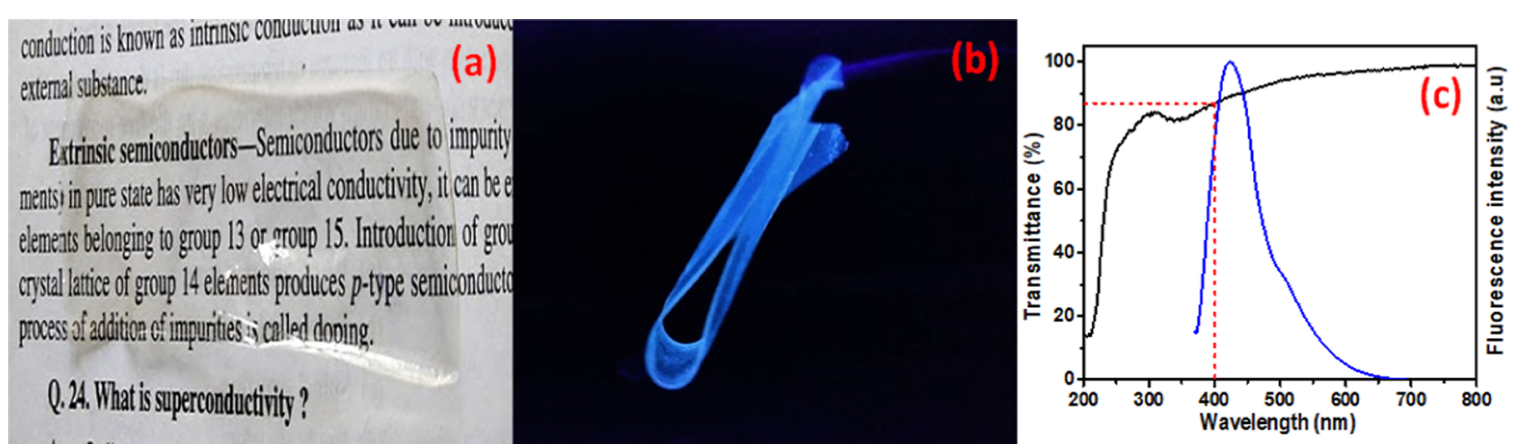

Figure 9. CBCDs/PVA film (a) under daylight, (b) under $365 \mathrm{~nm}$ UV light, and (c) corresponding transmittance spectra and fluorescence spectra under optimal excitation of $357 \mathrm{~nm}$.

fluorescent quenching is observed and provided output as 0 , while in the case of other inputs $(0 / 0,0 / 1,1 / 1)$, the output remains 1. The logic symbol, truth table, and output fluorescence intensity at $430 \mathrm{~nm}$ are shown in Figure 7.

Fluorescent Ink and Polymer. Finding novel applications to emerging materials like CDs is always important. Here, $\mathrm{CBCDs}$ possess unique properties, including strong and stable fluorescence and good transparency in the visible region, which makes them suitable candidates for fluorescent ink applications. ${ }^{63}$ To explore this, a sketch pen is filled with CBCDs aqueous solution (Figure S12) and employed for writing some text on commercial filter paper. As shown in Figure 8, the words "Osmania University" are clearly visible (showing blue fluorescence) under UV light, whereas the filter paper appeared as blank under daylight. It is worth mentioning that the information on the filter paper remained consistent and can be reproducible even after 30 days (when stored under ambient conditions) (Figure S13). These observations suggest that the $\mathrm{CBCDs}$ can be used as invisible ink for loading important information for secret communications and have a great potential for anticounterfeiting applications. Furthermore, with their high biocompatibility (low/nontoxic nature), CBCDs can be safely used as ink pads to form human fingerprints that do not contaminate the fingers. ${ }^{27}$ Figure $8 \mathrm{c}$ characterizes the CBCDs-formed fluorescent fingerprint on commercial filter paper, which could reflect human fingerprint clearly. Thus, the water-soluble CBCDs-based fluorescent ink could serve as an alternative to the traditional inks to form a clear, adelomorphic, long-lasting, and blue fluorescent fingerprint that can be easily cleaned with water and is pollution free. $^{64}$
Further extending their applicability to solid-state opticrelated fields is interesting and challenging. Efficient solid-state PL emission of CDs is extremely important for light-emitting diode (LED) applications. However, CDs in the solid state generally suffer from self-quenching because of the aggregation-caused quenching effect, which might be a result of excessive Forster resonance energy transfer among adjacent $\mathrm{CD}$ particles in the aggregated state or direct $\pi-\pi$ interaction. ${ }^{65}$ Dispersing CDs in the polymer matrix is considered to be an effective strategy to avoid this. Here, we have prepared solid-state films by dispersing CBCDs in poly(vinyl alcohol) (PVA). PVA was selected as polymer host because of its known high optical quality and desirable film properties. ${ }^{66}$ The as-fabricated films are highly transparent (about 90\% transmittance in the entire visible region) under daylight, while exhibited bright blue fluorescence under UV illumination (Figure 9). The absorption spectra (Figure S14a) of CBCDs in the polymer film is similar to those in solution, and the emission spectra (Figure S14b) also exhibited a similar excitation-dependent behavior.

In comparison to $\mathrm{CBCDs}$ aqueous solution, the optimal emission spectra of CBCDs/PVA film exhibited a blue shift of $6 \mathrm{~nm}$ and a slight red shift $(3 \mathrm{~nm})$ in the optimal excitation wavelength (Figure S15), which might be due to the fact that PVA environment is different from aqueous solution. ${ }^{67}$ The fluorescence QY of CBCDs in PVA matrix determined using QS as reference was found to be $33 \%$ (Table S8), which is higher than that in aqueous solution. This enhancement in the QY can be ascribed to the formation of hydrogen bonds between PVA and surface functional groups of CDs, which provide a stabilization effect on the electrons and holes for more efficient radiative recombination. ${ }^{63} \mathrm{~A}$ similar enhance- 
ment was observed in some previous reports also. ${ }^{63,66}$ Furthermore, the optical properties of CBCDs/PVA films are quite stable and no obvious decrease in PL emission is observed even after $24 \mathrm{~h}$ of continuous exposure to UV light (365 nm) (Figure S16). With improved and stable optical properties, CBCDs/PVA films have a great potential to serve as light conversion film/phosphor material in solid-state lighting systems.

\section{CONCLUSIONS}

In summary, we have successfully demonstrated a simple onestep hydrothermal method for the production of highly fluorescent $(\mathrm{QY}=26 \%) \mathrm{N}, \mathrm{S}$-codoped CDs from toxic CBs for the first time. Moderate reaction conditions, using waste as starting material, good production yield (9.6\%), and multifunctional nature of the formed products (CBCDs) make our method economical and scalable. The as-produced CBCDs were well characterized and explored for multiple applications. Bright and stable fluorescence coupled with high biocompatibility made CBCDs a potential candidate for bioimaging applications. They were successfully applied as "on-off-on" fluorescent probe for the sequential detection of $\mathrm{Fe}^{3+}$ and $\mathrm{AA}$ in real water and biological samples. CBCDs were further employed as biocompatible and invisible ink for loading data and forming fingerprints. The "visible-invisible" and "UVvisible" properties of CBCDs promise their applicability in anticounterfeiting. Furthermore, the CBCDs/PVA films with strong and stable emission might find direct or indirect applications in various optical/optoelectronic devices, ranging from fluorescent displays to LEDs.

\section{EXPERIMENTAL SECTION}

Materials. Cigarette butts were collected from the ash trays of a local bar and restaurant. All of the chemicals were of analytical grade and used without further purification. All solutions were prepared by using double-distilled (DD) water.

Synthesis of Carbon Dots. CBs ( $0.5 \mathrm{~g})$ were cut into small pieces (paper wrap was removed to avoid dust particles) and placed in a $100 \mathrm{~mL}$ of Erlenmeyer flask containing $20 \mathrm{~mL}$ of $40 \% \mathrm{H}_{2} \mathrm{SO}_{4}$ and sonicated for $1 \mathrm{~h}$. Then, the formed dispersion was transferred to a $25 \mathrm{~mL}$ Teflon-lined stainless steel autoclave and heated at $180{ }^{\circ} \mathrm{C}$ for $6 \mathrm{~h}$ in an electric oven. After cooling down to room temperature, the obtained solution was centrifuged to remove large particles. Then, the solution was neutralized with $\mathrm{Na}_{2} \mathrm{CO}_{3}$, centrifuged, and filtered through a $0.22 \mu \mathrm{m}$ syringe filter to remove the formed precipitate. Afterward, the solution was purified by dialyzing against DD water for $24 \mathrm{~h}$ using a dialysis tubing (molecular weight cut-off $3500 \mathrm{Da}$ ). Finally, CBCDs powder was obtained by freeze drying and used for the preparation of stock solution $\left(1 \mathrm{mg} \mathrm{mL}^{-1}\right)$.

Characterization. Fluorescence spectral measurements were carried out using a JASCO spectrofluorometer (FP8500 ) set with excitation and emission slit widths at $2.5 \mathrm{~nm}$. UV-vis spectral studies were conducted on a Shimadzu UVvis-NIR 3600 spectrophotometer. Fluorescence decay analysis was done on a Fluoro Cube, Lifetime System (Horiba Jobin Yvon) with a $370 \mathrm{~nm}$ Nano LED excitation source. Transmission electron microscopy (TEM) images were acquired on a JEOL 3010 microscope operating at an accelerating voltage of $200 \mathrm{kV}$ by drop-casting a proper dilution of $\mathrm{CBCDs}$ aqueous solution onto the carbon-coated copper grids. X-ray photoelectron spectroscopy (XPS) measurements were carried out by Kratos AXIS Ultra spectrometer with $\mathrm{Al} \mathrm{K \alpha} \mathrm{X}$-ray as the excitation source $(1486.71 \mathrm{eV})$. Fourier transform infrared (FTIR) spectrum was recorded on a Shimadzu IR Prestige-21 spectrophotometer. $\zeta$ potential measurements were conducted using a Malvern, Nano ZS90 Zetasizer. Raman spectra were measured with a Horiba Jobin Yvon LabRAM HR with a focal length of $800 \mathrm{~mm}$ and equipped with a $\mathrm{He}-\mathrm{Ne} 633 \mathrm{~nm}$ Laser. X-ray diffraction (XRD) patterns of CBCDs were obtained using an X'pert Pro powder X-ray diffractometer (the Netherlands) with $\mathrm{Cu} \mathrm{K} \alpha$ radiation, $\lambda=1.5406 \AA$.

Calculation of QY. QYs of all of the CBCD samples were calculated by using the slope method in which quinine sulfate (QS) is chosen as standard $(\Phi=54 \%)$. This particular method first involves the preparation of several concentrations of CBCDs aqueous solutions and QS $0.1 \mathrm{M} \mathrm{H}_{2} \mathrm{SO}_{4}$ solutions by maintaining the absorbance values less than 0.1 at their excitation wavelengths. Subsequently, the integrated emission intensities of all of the samples were recorded by exciting at $360 \mathrm{~nm}$. Afterward, the integrated emission intensities were plotted against corresponding absorbance values and the slope values of the obtained linear plots were computed. Finally, quantum yields were calculated by using the following equation

$$
\Phi_{\mathrm{x}}=\Phi_{\mathrm{st}}\left(\frac{\operatorname{Grad}_{x}}{\operatorname{Grad}_{s t}}\right)\left(\frac{\eta_{x}^{2}}{\eta_{s t}^{2}}\right)
$$

where $\Phi$ is the quantum yield, Grad is the gradient from the plot of integrated emission intensity versus absorbance, and $\eta$ is the refractive index of the solvent ( 1.33 for both solvents). The subscripts st and $\mathrm{x}$ denote standard (QS) and CBCDs, respectively.

Detection of $\mathrm{Fe}^{3+}$ and Ascorbic Acid. Solutions of all metal ions used in this experiment are prepared from corresponding salts in DD water. At room temperature, 1 $\mathrm{mL}$ of DD water is added to $1 \mathrm{~mL}$ of CBCDs aqueous solution $\left(0.1 \mathrm{mg} \mathrm{mL} \mathrm{m}^{-1}\right)$, the emission spectra of this sample are recorded at an excitation wavelength of $360 \mathrm{~nm}$, and the emission intensity at $430 \mathrm{~nm}$ is denoted as $F_{0}$. In a similar manner, $1 \mathrm{~mL}$ of different metal ion solutions of $200 \mu \mathrm{M}$ concentration were added to $1 \mathrm{~mL}$ of $\mathrm{CBCD}$ solution, shaken well, and incubated for $2 \mathrm{~min}$ and thereafter the emission intensity is recorded as F. A similar procedure was followed for the quantitative determination of $\mathrm{Fe}^{3+}$. In a typical assay, $1 \mathrm{~mL}$ of $\mathrm{CBCDs}$ solution was mixed with $1 \mathrm{~mL}$ of $\mathrm{Fe}^{3+}$ solution of varying concentrations. For the detection of ascorbic acid (AA), first, $0.5 \mathrm{~mL}$ of $\mathrm{Fe}^{3+}$ is added to $0.5 \mathrm{~mL}$ of CBCDs solution and then $1 \mathrm{~mL}$ of AA with varying concentrations was added. The final concentrations of $\mathrm{Fe}^{3+}$ and CBCDs are 100 $\mu \mathrm{M}$ and $0.05 \mathrm{mg} \mathrm{mL}^{-1}$, respectively. After $10 \mathrm{~min}$ of incubation, fluorescence response in recorded.

Cytotoxicity and Cellular Imaging. Standard MTT assay was employed for evaluating the in vitro cytotoxicity of cigarette butt-derived CDs. Experimental studies were performed on both normal (HEK-293) and cancerous (HeLa) cell lines. Complete details are provided in the Supporting Information.

Preparation of Fluorescent Films (CBCDs/PVA Composite Films). Poly(vinyl alcohol) (PVA) $(1 \mathrm{~g})$ is added to 10 $\mathrm{mL}$ of $\mathrm{DD}$ water under constant stirring, and the mixture was heated to dissolve PVA. After that, $1 \mathrm{~mL}$ of $1 \mathrm{mg} \mathrm{mL}^{-1}$ CBCDs 
aqueous solution was added to $9 \mathrm{~mL}$ of PVA solution and stirred gently for $10 \mathrm{~min}$. Finally, the mixture was drop-casted onto a clean glass slide and dried in an oven. After drying, the film was peeled off from the glass substrate to get a freestanding film.

\section{ASSOCIATED CONTENT}

\section{S Supporting Information}

The Supporting Information is available free of charge on the ACS Publications website at DOI: 10.1021/acsomega.8b01743.

Information as mentioned in the text; optimization of sulfuric acid; optimization of reaction conditions; comparison of synthetic conditions; SEM-EDX analysis; FTIR spectra; $\zeta$ potential; normalized emission spectra; plot of integrated fluorescence intensity vs absorbance; effect of ionic strength, $\mathrm{pH}, \mathrm{UV}$ irradiation, and temperature; long term storage stability; effect of various metal ions; optimization of $\mathrm{Fe}^{3+}$ detection conditions; comparison of analytical performance; optimization of AA detection; comparison of analytical performance for AA detection; real sample analysis; FTIR spectra of $\mathrm{CBCDs} / \mathrm{Fe}^{3+}$; percentage cell viability of HeLa and HEK-293 cells; sketch pen filled with CBCDs solution; reproducibility of information loaded with $\mathrm{CBCDs}$ fluorescent ink; optical properties of CBCDs/PVA film; quantum yield calculation of CBCDs/PVA film; photostability of CBCDs/PVA film (Tables S1-S8 and Figures $\mathrm{S} 1-\mathrm{S} 16)(\mathrm{PDF})$

\section{AUTHOR INFORMATION}

\section{Corresponding Author}

*E-mail: gvbhadram@gmail.com.

\section{ORCID $\odot$}

Veerabhadram Guttena: 0000-0003-2322-4333

\section{Notes}

The authors declare no competing financial interest.

\section{ACKNOWLEDGMENTS}

R.B. gratefully acknowledges CSIR-India for Senior Research Fellowship. The authors are grateful to MRC, MNIT Jaipur, for extending various analytical facilities. The authors acknowledge DST-FIST, New Delhi, India, for providing necessary analytical facilities in the department. Sincere thanks go to Department of Chemistry, Osmania University (OU), and CFRD-OU, for providing infrastructure and other necessary facilities.

\section{REFERENCES}

(1) World Health Organization. Tobacco and Its Environmental Impact: An Overview; WHO, 2017.

(2) Belhouari, Y.; Farnum, B.; Jenkins, C.; Kieser, J.; de Román, A. L.; McCauley, D.; Rochman, C.; Schreiber, R.; Schwartz, E.; Taylor, H. et al. Together for Our Ocean. International Coastal Cleanup 2017 Report, 2017.

(3) Slaughter, E.; Gersberg, R. M.; Watanabe, K.; Rudolph, J.; Stransky, C.; Novotny, T. E. Toxicity of Cigarette Butts, and Their Chemical Components, to Marine and Freshwater Fish. Tob. Control 2011, 20, i25-i29.

(4) Micevska, T.; Warne, M. S. J.; Pablo, F.; Patra, R. Variation in, and Causes of, Toxicity of Cigarette Butts to a Cladoceran and Microtox. Arch. Environ. Contam. Toxicol. 2006, 50, 205-212.
(5) Green, A. L. R.; Putschew, A.; Nehls, T. Littered Cigarette Butts as a Source of Nicotine in Urban Waters. J. Hydrol. 2014, 519, 34663474.

(6) Mohajerani, A.; Kadir, A. A.; Larobina, L. A Practical Proposal for Solving the World's Cigarette Butt Problem: Recycling in Fired Clay Bricks. Waste Manage. 2016, 52, 228-244.

(7) Mohajerani, A.; Tanriverdi, Y.; Nguyen, B. T.; Wong, K. K.; Dissanayake, H. N.; Johnson, L.; Whitfield, D.; Thomson, G.; Alqattan, E.; Rezaei, A. Physico-Mechanical Properties of Asphalt Concrete Incorporated with Encapsulated Cigarette Butts. Constr. Build. Mater. 2017, 153, 69-80.

(8) Lee, M.; Kim, G.-P.; Song, H. D.; Park, S.; Yi, J. Preparation of Energy Storage Material Derived from a Used Cigarette Filter for a Supercapacitor Electrode. Nanotechnology 2014, 25, No. 345601.

(9) Huang, F.; Xu, Y.; Peng, B.; Su, Y.; Jiang, F.; Hsieh, Y.-L.; Wei, Q. Coaxial Electrospun Cellulose-Core Fluoropolymer-Shell Fibrous Membrane from Recycled Cigarette Filter as Separator for High Performance Lithium-Ion Battery. ACS Sustainable Chem. Eng. 2015, 3, 932-940.

(10) Anmei, S.; Qingmei, Z.; Yuye, C.; Yilin, W. Preparation of Carbon Quantum Dots from Cigarette Filters and Its Application for Fluorescence Detection of Sudan I. Anal. Chim. Acta 2018, 1023, 115-120.

(11) Baker, S. N.; Baker, G. A. Luminescent Carbon Nanodots: Emergent Nanolights. Angew. Chem., Int. Ed. 2010, 49, 6726-6744.

(12) Lim, S. Y.; Shen, W.; Gao, Z. Carbon Quantum Dots and Their Applications. Chem. Soc. Rev. 2015, 44, 362-381.

(13) Himaja, A. L.; Karthik, P. S.; Singh, S. P. Carbon Dots: The Newest Member of the Carbon Nanomaterials Family. Chem. Rec. 2015, 15, 595-615.

(14) Hu, Y.; Zhang, L.; Li, X.; Liu, R.; Lin, L.; Zhao, S. Green Preparation of $S$ and $\mathrm{N}$ Co-Doped Carbon Dots from Water Chestnut and Onion as Well as Their Use as an Off-On Fluorescent Probe for the Quantification and Imaging of Coenzyme A. ACS Sustainable Chem. Eng. 2017, 5, 4992-5000.

(15) Sharma, V.; Tiwari, P.; Mobin, S. M. Sustainable Carbon-Dots: Recent Advances in Green Carbon Dots for Sensing and Bioimaging. J. Mater. Chem. B 2017, 5, 8904-8924.

(16) Pal, T.; Mohiyuddin, S.; Packirisamy, G. Facile and Green Synthesis of Multicolor Fluorescence Carbon Dots from Curcumin: In Vitro and in Vivo Bioimaging and Other Applications. ACS Omega 2018, 3, 831-843.

(17) Zheng, Y.; Xie, G.; Zhang, X.; Chen, Z.; Cai, Y.; Yu, W.; Liu, H.; Shan, J.; Li, R.; Liu, Y.; et al. Bioimaging Application and GrowthPromoting Behavior of Carbon Dots from Pollen on Hydroponically Cultivated Rome Lettuce. ACS Omega 2017, 2, 3958-3965.

(18) Tripathi, K. M.; Tran, T. S.; Tung, T. T.; Losic, D.; Kim, T. Water Soluble Fluorescent Carbon Nanodots from Biosource for Cells Imaging. J. Nanomater. 2017, 2017, 1-10.

(19) Feng, T.; Zeng, Q.; Lu, S.; Yan, X.; Liu, J.; Tao, S.; Yang, M.; Yang, B. Color-Tunable Carbon Dots Possessing Solid-State Emission for Full-Color Light-Emitting Diodes Applications. ACS Photonics 2018, 5, 502-510.

(20) Li, W.; Li, M.; Liu, Y.; Pan, D.; Li, Z.; Wang, L.; Wu, M. Three Minute Ultrarapid Microwave-Assisted Synthesis of Bright Fluorescent Graphene Quantum Dots for Live Cell Staining and White LEDs. ACS Appl. Nano Mater. 2018, 1, 1623-1630.

(21) Meziani, M. J.; Dong, X.; Zhu, L.; Jones, L. P.; Lecroy, G. E.; Yang, F.; Wang, S.; Wang, P.; Zhao, Y.; Yang, L.; et al. Visible-LightActivated Bactericidal Functions of Carbon "Quantum" Dots. ACS Appl. Mater. Interfaces 2016, 8, 10761-10766.

(22) Chen, J.; Wei, J. S.; Zhang, P.; Niu, X. Q.; Zhao, W.; Zhu, Z. Y.; Ding, H.; Xiong, H. M. Red-Emissive Carbon Dots for Fingerprints Detection by Spray Method: Coffee Ring Effect and Unquenched Fluorescence in Drying Process. ACS Appl. Mater. Interfaces 2017, 9, 18429-18433.

(23) Duarah, R.; Karak, N. Facile and Ultrafast Green Approach to Synthesize Biobased Luminescent Reduced Carbon Nanodot: An 
Efficient Photocatalyst. ACS Sustainable Chem. Eng. 2017, 5, 94549466.

(24) Singh, A.; Khare, P.; Verma, S.; Bhati, A.; Sonker, A. K.; Tripathi, K. M.; Sonkar, S. K. Pollutant Soot for Pollutant Dye Degradation: Soluble Graphene Nanosheets for Visible Light Induced Photodegradation of Methylene Blue. ACS Sustainable Chem. Eng. 2017, 5, 8860-8869.

(25) Tripathi, K. M.; Tran, T. S.; Kim, Y. J.; Kim, T. Green Fluorescent Onion-Like Carbon Nanoparticles from Flaxseed Oil for Visible Light Induced Photocatalytic Applications and Label-Free Detection of Al(III) Ions. ACS Sustainable Chem. Eng. 2017, 5, 39823992.

(26) Briscoe, J.; Marinovic, A.; Sevilla, M.; Dunn, S.; Titirici, M. Biomass-Derived Carbon Quantum Dot Sensitizers for Solid-State Nanostructured Solar Cells. Angew. Chem., Int. Ed. 2015, 54, 44634468.

(27) Qu, S.; Wang, X.; Lu, Q.; Liu, X.; Wang, L. A Biocompatible Fluorescent Ink Based on Water-Soluble Luminescent Carbon Nanodots. Angew. Chem., Int. Ed. 2012, 51, 12215-12218.

(28) Zuo, G.; Xie, A.; Pan, X.; Su, T.; Li, J.; Dong, W. FluorineDoped Cationic Carbon Dots for E Ffi Cient Gene Delivery. ACS Appl. Nano Mater. 2018, 1, 2376-2385.

(29) Zhang, X.; Jiang, M.; Niu, N.; Chen, Z.; Li, S.; Liu, S.; Li, J. Natural-Product-Derived Carbon Dots: From Natural Products to Functional Materials. ChemSusChem 2018, 11, 11-24.

(30) Yang, Z.-C.; Wang, M.; Yong, A. M.; Wong, S. Y.; Zhang, X.-H.; Tan, H.; Chang, A. Y.; Li, X.; Wang, J. Intrinsically Fluorescent Carbon Dots with Tunable Emission Derived from Hydrothermal Treatment of Glucose in the Presence of Monopotassium Phosphate. Chem. Commun. 2011, 47, 11615.

(31) Zhuang, Q.; Cao, W.; Ni, Y.; Wang, Y. Synthesis-Identification Integration: One-Pot Hydrothermal Preparation of Fluorescent Nitrogen-Doped Carbon Nanodots for Differentiating Nucleobases with the Aid of Multivariate Chemometrics Analysis. Talanta 2018, 185, 491-498.

(32) Zhuang, Q.; Guo, P.; Zheng, S.; Lin, Q.; Lin, Y.; Wang, Y.; Ni, Y. Green Synthesis of Luminescent Graphitic Carbon Nitride Quantum Dots from Human Urine and Its Bioimaging Application. Talanta 2018, 188, 35-40.

(33) Zhuang, Q.; Wang, Y.; Ni, Y. Solid-Phase Synthesis of Graphene Quantum Dots from the Food Additive Citric Acid under Microwave Irradiation and Their Use in Live-Cell Imaging. Luminescence 2016, 31, 746-753.

(34) Wang, Y.; Zhuang, Q.; Ni, Y. Facile Microwave-Assisted SolidPhase Synthesis of Highly Fluorescent Nitrogen-Sulfur-Codoped Carbon Quantum Dots for Cellular Imaging Applications. Chem. Eur. J. 2015, 21, 13004-13011.

(35) Zhu, H.; Wang, X.; Li, Y.; Wang, Z.; Yang, F.; Yang, X. Microwave Synthesis of Fluorescent Carbon Nanoparticles with Electrochemiluminescence Properties. Chem. Commun. 2009, 5118.

(36) Wang, Y.; Hu, A. Carbon Quantum Dots: Synthesis, Properties and Applications. J. Mater. Chem. C 2014, 2, 6921.

(37) Kargbo, O.; Jin, Y.; Ding, S. Recent Advances in Luminescent Carbon Dots. Curr. Anal. Chem. 2014, 11, 4-21.

(38) Park, S. Y.; Lee, H. U.; Park, E. S.; Lee, S. C.; Lee, J.-W.; Jeong, S. W.; Kim, C. H.; Lee, Y.-C.; Huh, Y. S.; Lee, J. Photoluminescent Green Carbon Nanodots from Food-Waste-Derived Sources: LargeScale Synthesis, Properties, and Biomedical Applications. ACS Appl. Mater. Interfaces 2014, 6, 3365-3370.

(39) Purkayastha, M. D.; Manhar, A. K.; Das, V. K.; Borah, A.; Mandal, M.; Thakur, A. J.; Mahanta, C. L. Antioxidative, Hemocompatible, Fluorescent Carbon Nanodots from an "End-ofPipe" Agricultural Waste: Exploring Its New Horizon in the FoodPackaging Domain. J. Agric. Food Chem. 2014, 62, 4509-4520.

(40) Jeong, Y.; Moon, K.; Jeong, S.; Koh, W. G.; Lee, K. Converting Waste Papers to Fluorescent Carbon Dots in the Recycling Process without Loss of Ionic Liquids and Bioimaging Applications. ACS Sustainable Chem. Eng. 2018, 6, 4510-4515.
(41) Hu, Y.; Yang, J.; Tian, J.; Jia, L.; Yu, J.-S. Waste Frying Oil as a Precursor for One-Step Synthesis of Sulfur-Doped Carbon Dots with PH-Sensitive Photoluminescence. Carbon 2014, 77, 775-782.

(42) Xu, Y.; Li, D.; Liu, M.; Niu, F.; Liu, J.; Wang, E. EnhancedQuantum Yield Sulfur/Nitrogen Co-Doped Fluorescent Carbon Nanodots Produced from Biomass Enteromorpha Prolifera: Synthesis, Posttreatment, Applications and Mechanism Study. Sci. Rep. 2017, 7, No. 4499.

(43) Dong, Y.; Pang, H.; Yang, H. B.; Guo, C.; Shao, J.; Chi, Y.; Li, C. M.; Yu, T. Carbon-Based Dots Co-Doped with Nitrogen and Sulfur for High Quantum Yield and Excitation-Independent Emission. Angew. Chem., Int. Ed. 2013, 52, 7800-7804.

(44) Hu, X.; Xu, C.; Gao, J.; Yang, G.; Geng, C.; Chen, F.; Fu, Q. Toward Environment-Friendly Composites of Poly(Propylene Carbonate) Reinforced with Cellulose Nanocrystals. Compos. Sci. Technol. 2013, 78, 63-68.

(45) Fu, M.; Ehrat, F.; Wang, Y.; Milowska, K. Z.; Reckmeier, C.; Rogach, A. L.; Stolarczyk, J. K.; Urban, A. S.; Feldmann, J. Carbon Dots: A Unique Fluorescent Cocktail of Polycyclic Aromatic Hydrocarbons. Nano Lett. 2015, 15, 6030-6035.

(46) Yang, G.; Wan, X.; Su, Y.; Zeng, X.; Tang, J. Acidophilic SDoped Carbon Quantum Dots Derived from Cellulose Fibers and Their Fluorescence Sensing Performance for Metal Ions in an Extremely Strong Acid Environment. J. Mater. Chem. A 2016, 4, 12841-12849.

(47) Miao, X.; Yan, X.; Qu, D.; Li, D.; Tao, F. F.; Sun, Z. Red Emissive Sulfur, Nitrogen Codoped Carbon Dots and Their Application in Ion Detection and Theraonostics. ACS Appl. Mater. Interfaces 2017, 9, 18549-18556.

(48) Peng, J.; Gao, W.; Gupta, B. K.; Liu, Z.; Romero-Aburto, R.; Ge, L.; Song, L.; Alemany, L. B.; Zhan, X.; Gao, G.; et al. Graphene Quantum Dots Derived from Carbon Fibers. Nano Lett. 2012, 12, 844-849.

(49) Liu, X.; Li, H.-B.; Shi, L.; Meng, X.; Wang, Y.; Chen, X.; Xu, H.; Zhang, W.; Fang, X.; Ding, T. Structure and Photoluminescence Evolution of Nanodots during Pyrolysis of Citric Acid: From Molecular Nanoclusters to Carbogenic Nanoparticles. J. Mater. Chem. C 2017, 5, 10302-10312.

(50) Ramanan, V.; Thiyagarajan, S. K.; Raji, K.; Suresh, R.; Sekar, R.; Ramamurthy, P. Outright Green Synthesis of Fluorescent Carbon Dots from Eutrophic Algal Blooms for In Vitro Imaging. ACS Sustainable Chem. Eng. 2016, 4, 4724-4731.

(51) Ramanan, V.; Siddaiah, B.; Raji, K.; Ramamurthy, P. Green Synthesis of Multifunctionalized, Nitrogen-Doped, Highly Fluorescent Carbon Dots from Waste Expanded Polystyrene and Its Application in the Fluorimetric Detection of $\mathrm{Au}^{3+}$ Ions in Aqueous Media. ACS Sustainable Chem. Eng. 2018, 6, 1627-1638.

(52) van Dam, B.; Nie, H.; Ju, B.; Marino, E.; Paulusse, J. M. J.; Schall, P.; Li, M.; Dohnalová, K. Excitation-Dependent Photoluminescence from Single-Carbon Dots. Small 2017, 13, No. 1702098.

(53) Dong, Y.; Wang, R.; Li, G.; Chen, C.; Chi, Y.; Chen, G. Polyamine-Functionalized Carbon Quantum Dots as Fluorescent Probes for Selective and Sensitive Detection of Copper Ions. Anal. Chem. 2012, 84, 6220-6224.

(54) Bandi, R.; Dadigala, R.; Gangapuram, B. R.; Guttena, V. Green Synthesis of Highly Fluorescent Nitrogen - Doped Carbon Dots from Lantana Camara Berries for Effective Detection of Lead(II) and Bioimaging. J. Photochem. Photobiol., B 2018, 178, 330-338.

(55) Sachdev, A.; Gopinath, P. Green Synthesis of Multifunctional Carbon Dots from Coriander Leaves and Their Potential Application as Antioxidants, Sensors and Bioimaging Agents. Analyst 2015, 140, $4260-4269$

(56) Xu, H.; Yang, X.; Li, G.; Zhao, C.; Liao, X. Green Synthesis of Fluorescent Carbon Dots for Selective Detection of Tartrazine in Food Samples. J. Agric. Food Chem. 2015, 63, 6707-6714.

(57) Lakowicz, J. R. Principles of Fluorescence Spectroscopy Principles of Fluorescence Spectroscopy; Springer, 2006. 
(58) Hong, G.; Diao, S.; Antaris, A. L.; Dai, H. Carbon Nanomaterials for Biological Imaging and Nanomedicinal Therapy. Chem. Rev. 2015, 115, 10816-10906.

(59) Liao, J.; Cheng, Z.; Zhou, L. Nitrogen-Doping Enhanced Fluorescent Carbon Dots: Green Synthesis and Their Applications for Bioimaging and Label-Free Detection of $\mathrm{Au}^{3+}$ Ions. ACS Sustainable Chem. Eng. 2016, 4, 3053-3061.

(60) Kang, Y.-F.; Fang, Y.-W.; Li, Y.-H.; Li, W.; Yin, X.-B. NucleusStaining with Biomolecule-Mimicking Nitrogen-Doped Carbon Dots Prepared by a Fast Neutralization Heat Strategy. Chem. Commun. 2015, 51, 16956-16959.

(61) Gao, G.; Jiang, Y.-W.; Jia, H.-R.; Yang, J.; Wu, F.-G. On-off-on Fluorescent Nanosensor for $\mathrm{Fe}^{3+}$ Detection and Cancer/Normal Cell Differentiation via Silicon-Doped Carbon Quantum Dots. Carbon 2018, 134, 232-243.

(62) Sharma, V.; Kaur, N.; Tiwari, P.; Saini, A. K.; Mobin, S. M. Multifunctional Fluorescent "Off-On-Off" Nanosensor for $\mathrm{Au}^{3+}$ and $\mathrm{S}^{2-}$ Employing N-S Co-Doped Carbon-dots. Carbon 2018, 139, 393-403.

(63) Liu, Y.; Zhou, L.; Li, Y.; Deng, R.; Zhang, H. Highly Fluorescent Nitrogen-Doped Carbon Dots with Excellent Thermal and Photo Stability Applied as Invisible Ink for Loading Important Information and Anti-Counterfeiting. Nanoscale 2017, 9, 491-496.

(64) Lu, W.; Gong, X.; Yang, Z.; Zhang, Y.; Hu, Q.; Shuang, S.; Dong, C.; Choi, M. M. F. High-Quality Water-Soluble Luminescent Carbon Dots for Multicolor Patterning, Sensors, and Bioimaging. RSC Adv. 2015, 5, 16972-16979.

(65) Zheng, J.; Wang, Y.; Zhang, F.; Yang, Y.; Liu, X.; Guo, K.; Wang, H.; Xu, B. Microwave-Assisted Hydrothermal Synthesis of Solid-State Carbon Dots with Intensive Emission for White LightEmitting Devices. J. Mater. Chem. C 2017, 5, 8105-8111.

(66) Liu, Y.; Wang, P.; Fernando, K. A. S.; LeCroy, G. E.; Maimaiti, H.; Harruff-Miller, B. A.; Lewis, W. K.; Bunker, C. E.; Hou, Z.-L.; Sun, Y.-P. Enhanced Fluorescence Properties of Carbon Dots in Polymer Films. J. Mater. Chem. C 2016, 4, 6967-6974.

(67) Wang, Y.; Zhao, Y.; Zhang, F.; Chen, L.; Yang, Y.; Liu, X. Fluorescent Polyvinyl Alcohol Films Based on Nitrogen and Sulfur Co-Doped Carbon Dots towards White Light-Emitting Devices. New J. Chem. 2016, 40, 8710-8716. 